\title{
Kybernetika
}

\section{Petr Mandl}

\section{Elements of stochastic analysis}

Kybernetika, Vol. 14 (1978), No. Suppl1, (1),3--54

Persistent URL: http://dml.cz/dmlcz/125509

\section{Terms of use:}

(C) Institute of Information Theory and Automation AS CR, 1978

Institute of Mathematics of the Academy of Sciences of the Czech Republic provides access to digitized documents strictly for personal use. Each copy of any part of this document must contain these Terms of use.

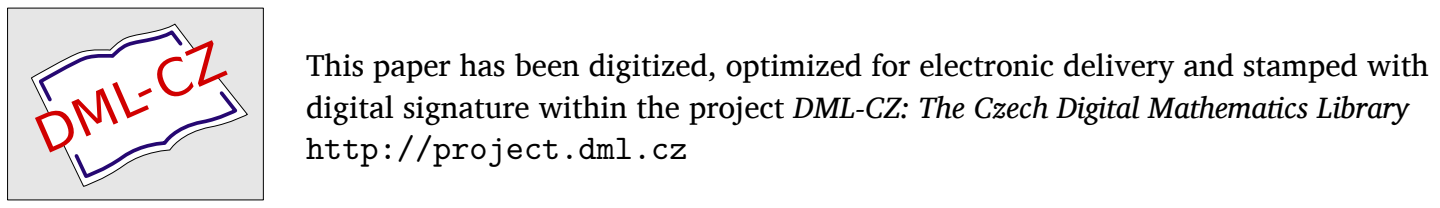




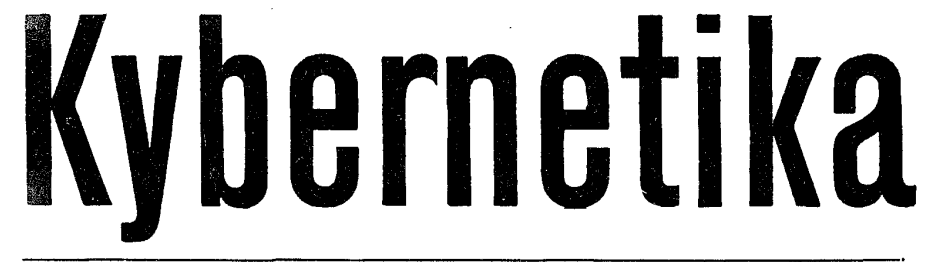

Elements of Stochastic Analysis

Petr Mandl

ACADEMIA

PRAHA 


\section{INTRODUCTION}

N. Wiener, A. Z. Zygmunt, and R. E. A. C. Paley introduced the integrals of nonrandom functions with respect to the Brownian motion process in 1933. K. Itô developed the theory of stochastic integrals and stochastic differential equations, and discovered the renowned Itô formula $(1944,1946)$. Since then, stochastic analysis has been perfectioned into a theory of considerable extent and completeness. Much of its growths has been stimulated by the interest, it receives from the engineers working in filtering theory, in control theory or in electronics, as well as from the physicists, economists, and biologists, dealing with phenomena in which the random noise plays an important role. Simultaneously to the "stochastization" of their disciplines the applied scientists become familiar with more advanced parts of probability theory. This positive trend is often incorrectly reflected in textbooks, where refined mathematical notions are subject to rather crude arguments and unnecessary recourses to the intuition. The aim of this text is to keep uniformly the presentation on a logical level bellow which a real understanding of stochastic analysis is hardly possible. The author is aware that the main appeal of stochastic analysis lies for applied scientists in the calculus it contains. I.e. in a method how to arrive to conclusions by means of calculating differentials, solving equations, and handling formulas. To stress that, the text will be followed by a separate collection of problems Exercises in Stochastic Analysis prepared jointly with V. Lánská and I. Vrkoč.

Definitions recalled in Section 1.1 show the knowledge of probability theory expected from the reader. It corresponds, e.g., to M. Rosenblatt's Random Processes, Graduate Text in Mathematics 17, Springer-Verlag. The present text covers the integration, the differentiation, and stochastic differential equations. Filtering theory, control theory or statistics of random processes are not included. They are autonomous disciplines using stochastic analysis.

Chapters $1,2, \ldots$, are divided into sections $\S 1.1, \S 1.2, \ldots$ Reference to Theorem 2.1 recalls the first theorem of Chapter 2. Inside that chapter it is reference to Theorem 1. Standard mathematical symbols are used. The meaning of the less frequent ones is as follows:

a.s. almost surely

const. finite positive constant

1.i.m. limit in mean square

plim limit in probability

$R^{m} \quad m$-dimensional Euclidean space

$\chi_{A} \quad$ indicator function of $A$ 
$\sigma a() \sigma$-algebra generated by random events or random processes in the brackets

[a] integer part of $a$, where square bracket not plausible

$a \wedge b \min (a, b)$

, transposition of vectors and matrices

end of proof or example

\section{THE WIENER PROCESS}

\subsection{Basic Notions}

The mathematical structure for a probabilistic theory is a triple $(\Omega, \mathscr{A}, P)$ called probability space. $\Omega$ is the set of elementary events, $\mathscr{A}$ is the $\sigma$-algebra of random events, and $P$ is a probability measure on $\mathscr{A}$. Random variables $\xi(\omega), \eta(\omega), \ldots$, are $\mathscr{A}$-measurable functions on $\Omega$. We shall mostly write only $\xi, \eta, \ldots$ Integration with respect to $P$ gives the mathematical expectation

$$
E \xi=\int \xi \mathrm{d} P
$$

provided that the integral on the right-hand side exists.

Next we introduce conditional expectations. Let $\xi, \eta$ be random variables on $(\Omega, \mathscr{A}, P), E|\xi|<\infty$. Assume that their joint probability distribution has density $f(x, y)$. Let

$$
f_{2}(y)=\int_{-\infty}^{\infty} f(x, y) \mathrm{d} x>0, \quad y \in(-\infty, \infty) .
$$

The conditional expectation of $\xi$ given $\eta=y$ equals

$$
\int_{-\infty}^{\infty} x f(x, y) \mathrm{d} x / f_{2}(y)=e(y), \quad y \in(-\infty, \infty) .
$$

It is practical to transfer the conditional expectation to $(\Omega, \mathscr{A}, P)$ and to define $\varepsilon(\omega)=e(\eta(\omega))$. Random variable $\varepsilon$ is the expectation of $\xi$ after the observation of $\eta$. Let us point out the characteristic properties of $\varepsilon$. Let $B$ be a Borel set in $(-\infty, \infty)$, $A=\{\omega: \eta \in B\}$. Then

$$
\begin{gathered}
\int_{A} \varepsilon \mathrm{d} P=\int_{B} e(y) f_{2}(y) \mathrm{d} y=\int_{-\infty}^{\infty} \int_{B} x f(x, y) \mathrm{d} x \mathrm{~d} y= \\
=\iint \chi_{B}(y) x f(x, y) \mathrm{d} x \mathrm{~d} y=E \chi_{B}(\eta) \xi=\int_{A} \xi \mathrm{d} P .
\end{gathered}
$$

\section{4}


$\chi_{B}$ is the indicator of $B$. Random events $\{\eta \in B\}, B$ Borelian, form a $\sigma$-algebra $\mathscr{C}=$ $=\sigma a(\eta) \subset \mathscr{A} . \mathscr{C}$ is the least $\sigma$-algebra with respect to which $\eta$ is measurable. $\varepsilon$ is $\mathscr{C}$-measurable, since

$$
\{\varepsilon \in B\}=\left\{\eta \in e^{-1}(B)\right\} \in \sigma a(\eta) .
$$

This together with (1) motivates the general definition of conditional expectation.

Definition 1. Let $\xi$ be a random variable on $(\Omega, \mathscr{A}, P), E|\xi|<\infty$, let $\mathscr{C}$ be a $\sigma$-algebra $\mathscr{C} \subset \mathscr{A}$. We call conditional expectation of $\xi$ with respect to $\mathscr{C}$ a random variable $E\{\xi \mid \mathscr{C}\}$, which is $\mathscr{C}$-measurable, and

$$
\int_{A} E\{\xi \mid \mathscr{C}\} \mathrm{d} P=\int_{A} \xi \mathrm{d} P \text { for } A \in \mathscr{C} .
$$

(2) determines $E\{\xi \mid \mathscr{C}\}$ up to a set of probability 0 . Frequently the definition is extended to include $\mathscr{C}$-nonmeasurable random variables equal to $E\{\xi \mid \mathscr{C}\}$ a.s. (almost surely).

Theorem 1. The conditional expectation exists provided $E|\xi|<\infty$.

Proof. $Q(A)=\int_{A} \xi \mathrm{d} P, A \in \mathscr{C}$, is a finite signed measure on $\mathscr{C}$, absolutely continuous with respect to $P$. In virtue on the Random-Nikodym theorem

$$
Q(A)=\int_{A} \varepsilon \mathrm{d} P, \quad A \in \mathscr{C},
$$

where $\varepsilon=\mathrm{d} Q / \mathrm{d} P$ is $\mathscr{C}$-measurable. We set $E\{\xi \mid \mathscr{C}\}=\varepsilon$.

The conditional probability of $A \in \mathscr{A}$ with respect to $\mathscr{C}$

$$
P(A \mid \mathscr{C})=E\left\{\chi_{A} \mid \mathscr{C}\right\} \text {. }
$$

If $\mathscr{C}$ is generated by disjoint events $C_{1}, \ldots, C_{n}$,

$$
P\left(C_{k}\right)>0, \quad k=1, \ldots, n, \bigcup_{k=1}^{n} C_{k}=\Omega,
$$

then

$$
P(A \mid \mathscr{C})=\sum_{k=1}^{n} \chi_{C_{k}} P\left(A \cap C_{k}\right) P\left(C_{k}\right)^{-1}=\sum_{k=1}^{n} \chi_{C_{k}} P\left(A \mid C_{k}\right)
$$

Let us recall some properties of conditional expectations.

Property 1. Let $E|\xi|<\infty, E|\eta|<\infty$. Then

$$
E\{\xi+\eta \mid \mathscr{C}\}=E\{\xi \mid \mathscr{C}\}+E\{\eta \mid \mathscr{C}\} .
$$

We should have written a.s. in (3). But we shall regularly omit this. 
Property 2. Let $E|\xi|<\infty, \eta$ be $\mathscr{C}$-measurable, $E|\eta \xi|<\infty$. Then

$$
E\{\eta \xi \mid \mathscr{C}\}=\eta E\{\xi \mid \mathscr{C}\}
$$

Proof. If $\eta=\chi_{c}, C \in \mathscr{B}$, then from (2)

(4) $\int_{A} \eta E\{\xi \mid \mathscr{C}\} \mathrm{d} P=\int_{A \cap C} E\{\xi \mid \mathscr{C}\} \mathrm{d} P=\int_{A \cap C} \xi \mathrm{d} P=\int_{A} \eta \xi \mathrm{d} P$ for $A \in \mathscr{C}$.

(4) is extended to

$$
\eta=\sum_{k=1}^{n} b_{k} \chi_{c_{k}}, \quad C_{k} \in \mathscr{C}, \quad b_{k} \text { constants, } \quad k=1, \ldots, n,
$$

and then it is established for general $\eta$ by a standard approximation procedure.

Property 3. Let $E|\xi|<\infty$. Then

$$
|E\{\xi \mid \mathscr{C}\}| \leqq E\{|\xi| \mid \mathscr{C}\} \text {. }
$$

Proof. From $|\xi| \pm \xi \geqq 0$ follows

$$
0 \leqq E\{|\xi| \pm \xi \mid \mathscr{C}\}=E\{|\xi| \mid \mathscr{C}\} \pm E\{\xi \mid \mathscr{C}\}
$$

Property 4. Let $E|\xi|<\infty$, and let $\mathscr{C}_{1} \subset \mathscr{C}_{2} \subset \mathscr{A}$ be $\sigma$-algebras. Then

$$
E\left\{E\left\{\xi \mid \mathscr{C}_{2}\right\} \mid \mathscr{C}_{1}\right\}=E\left\{\xi \mid \mathscr{C}_{1}\right\} .
$$

Proof. We have to verify

$$
\int_{A} E\left\{\xi \mid \mathscr{C}_{1}\right\} \mathrm{d} P=\int_{A} E\left\{\xi \mid \mathscr{C}_{2}\right\} \mathrm{d} P \text { for } A \in \mathscr{C}_{1} .
$$

This is true, since both sides are equal to $\int_{A} \xi \mathrm{d} P$ by the definition of conditional expectation.

A particular case of $(5)$ is

$$
E E\{\xi \mid \mathscr{C}\}=E \xi
$$

Property 5. Let $E \xi^{2}<\infty, \eta$ be $\mathscr{C}$-measurable, $E \eta^{2}<\infty$. Then

$$
E(\xi-E\{\check{\zeta} \mid \mathscr{C}\})^{2} \leqq E(\xi-\eta)^{2} .
$$

Consequently, $E\{\xi \mid \mathscr{C}\}$ is in the mean square the best prediction of $\xi$ based on $\mathscr{C}$ 
Proof.

(7)

$$
\begin{gathered}
E\left\{(\xi-\eta)^{2} \mid \mathscr{C}\right\}=E\left\{(\xi-E\{\xi \mid \mathscr{C}\})^{2} \mid \mathscr{C}\right\}+ \\
+E\left\{(E\{\xi \mid \mathscr{C}\}-\eta)^{2} \mid \mathscr{C}\right\}+2(E\{\xi \mid \mathscr{C}\}-\eta) E\{\xi-E\{\xi \mid \mathscr{C}\} \mid \mathscr{C}\} .
\end{gathered}
$$

The last term equals 0 , and hence

$$
E\left\{(\xi-\eta)^{2} \mid \mathscr{C}\right\} \geqq E\left\{(\xi-E\{\xi \mid \mathscr{C}\})^{2} \mid \mathscr{C}\right\} .
$$

From here (6) follows by taking mathematical expectation of both sides.

Property 6. Let $E \xi^{2}<\infty$. Then

$$
E\left\{\xi^{2} \mid \mathscr{C}\right\} \geqq(E\{\xi \mid \mathscr{C}\})^{2}
$$

Proof. From (7) for $\eta \equiv 0$,

$$
E\left\{\xi^{2} \mid \mathscr{C}\right\} \geqq E\left\{(E\{\xi \mid \mathscr{C}\})^{2} \mid \mathscr{C}\right\}=(E\{\xi \mid \mathscr{C}\})^{2} .
$$

(8) is a special case of Jensen's inequality. Let $E|\xi|<\infty$, and let $f(x)$ be a convex function on $(-\infty, \infty), E|f(\xi)|<\infty$. Then

$$
E\{f(\xi) \mid \mathscr{C}\} \geqq f(E\{\xi \mid \mathscr{C}\}) .
$$

Consider now the notion of independence. Random event $A \in \mathscr{A}$ is independent of (the events from) $\mathscr{C}$, if

$$
P(A \mid \mathscr{C})=P(A)
$$

From the definition of conditional probability, for $C \in \mathscr{C}$,

$$
P(A \cap C)=\int_{C} \chi_{A} \mathrm{~d} P=\int_{C} P(A \mid \mathscr{C}) \mathrm{d} P=P(A) \int_{C} \mathrm{~d} P=P(A) P(C) .
$$

Random variable $\xi$ is independent of $\mathscr{C}$, if random events $\{\xi \in B\}, B$ Borel set, are independent of $\mathscr{C}$. It holds then $E\{\xi \mid \mathscr{C}\}=E \xi$ provided that $E|\xi|<\infty$.

A random process (random function) is a family of random variables

$$
X=\left\{X_{r}(\omega), t \in J\right\} .
$$

$J$ will be here an interval $([0, \infty),[0, T]$, etc.), $t$ will be viewed as time parameter. For $\omega \in \Omega, X_{t}(\omega)$ as function of $t$ is called the trajectory of $X$. Random processes are often defined by determining their joint probability distributions

$$
\begin{gathered}
P\left(X_{t_{1}} \leqq x_{1}, \quad X_{t_{2}} \leqq x_{2}, \ldots, X_{t_{n}} \leqq x_{n}\right), t_{1}, \ldots, t_{n} \in J, \\
x_{1}, \ldots, x_{n} \in[-\infty, \infty] .
\end{gathered}
$$


If the distributions (9) fulfil obvious symmetry and consistency conditions, then by a theorem of $\mathrm{A}$. N. Kolmogorov there exists a probability space $(\Omega, \mathscr{A}, P)$ carrying a family $X=\left\{X_{t}, t \in J\right\}$ of random variables the joint distributions of which coincide with (9).

Random processes considered in this text will be assumed measurable.

Definition 2. Random process $X=\left\{X_{t}(\omega), t \in J\right\}$ on $(\Omega, \mathscr{A}, P)$ is called measurable, if $X_{t}(\omega)$ is a $\mathscr{B}_{J} \times \mathscr{A}$ - measurable function of $(t, \omega)$ on $J \times \Omega$. $\mathscr{B}_{J}$ are the Borel sets in $J$.

The measurability assumption is of technical nature, and has the following consequences.

1. For each $\omega$ the trajectory $X_{t}(\omega)$ is a Borel measurable function of $t$.

2. The Fubini theorem is applicable,

$$
\int_{J \times \Omega} X_{t}(\omega) \mathrm{d} t \times \mathrm{d} P=\int_{J} E X_{t} \mathrm{~d} t=E \int_{J} X_{t} \mathrm{~d} t
$$

provided that the double integral exists. In particular, $\int_{J} X_{t} \mathrm{~d} t$ is defined and $\mathscr{A}$-measurable outside a set of probability 0 .

Theorem 2. A random process $X=\left\{X_{t}, t \in J\right\}$ with trajectories continuous from the right (from the left) is measurable.

Proof. Assume $X$ to be left-continuous. Define, for $n=1,2, \ldots$,

$$
{ }^{n} X_{t}=X_{k / n} \text { for } t \in\left[\frac{k}{n}, \frac{k+1}{n}\right) \cap J, \quad k=0,1, \ldots
$$

Processes ${ }^{n} X$ are measurable since for any Borel set $B$

$$
\left\{(t, \omega):{ }^{n} X_{t}(\omega) \in B\right\}=\bigcup_{k=0}^{\infty}\left(\left[\frac{k}{n}, \frac{k+1}{n}\right) \cap J\right) \times\left\{\omega: X_{k / n}(\omega) \in B\right\} \in \mathscr{B}_{J} \times \mathscr{A} .
$$

By the left-continuity $\lim ^{n} X_{t}=X_{t}$. Hence, $X$ is measurable. For $X$ right-continuous the proof is similar.

The operations of stochastic calculus mostly determine random processes only up to events of probability 0 . The same holds, e.g., about $\int_{J} X_{t} \mathrm{~d} t$ in (10). It is obvious to consider random processes (random variables) coinciding outside an event of probability 0 as indistinguishable, and we shall make use of this convention. A random process, say $X$, will be assumed to have any property which has almost surely a process indistinguishable of $X$. For example, if $X$ is defined and continuous almost 
surely, it is indistinguishable from a continuous process $X^{*}$. By Theorem $2, X^{*}$ is measurable. Hence, $X$ will be called measurable, and will be represented by $X^{*}$ in reasonings, where this property matters.

The above said extends in a straight-forward way to vector valued random variables $\xi=\left(\xi^{1}, \ldots, \xi^{m}\right), \ldots$, and to vector valued random processes $\left\{\bar{X}_{t}=\left(X_{t}^{1}, \ldots, X_{t}^{m}\right)^{\prime}\right.$, $t \in J\}$. The prime denotes the transposition into column vectors. In the sequel, random variables defined without further specification will be real valued with the exception of stopping times taking on also $+\infty$.

\subsection{The Wiener Process}

Without refering to it explicitely, we assume a probability space $(\Omega, \mathscr{A}, P)$ to carry the random variables and processes we are going to define.

Definition 3. We call Wiener process a random process $W=\left\{W_{t}, t \geqq 0\right\}$ with the following properties:

1. For $t \geqq 0, s>0$, the increments $W_{t+s}-W_{t}$ have normal distribution with mean 0 and variance $c s(c>0)$.

2. For arbitrary times $0 \leqq t_{1}<t_{2}<\ldots<t_{n}$ the increments

$$
W_{t_{2}}-W_{t_{1}}, \quad W_{t_{3}}-W_{t_{2}}, \ldots, W_{t_{n}}-W_{t_{n-1}}
$$

are mutually independent random variables.

3. The trajectories of $W$ are continuous (almost surely).

Unless stated otherwise, we shall set $c=1, W_{0} \equiv 0$ (means $W_{0}=0$ a.s.).

1 and 2 are compatible. The probability distribution, which results from adding the increments is normal with mean 0 and variance

$$
\operatorname{Var}\left(W_{t_{n}}-W_{t_{1}}\right)=\sum_{k=1}^{n-1} \operatorname{Var}\left(W_{t_{k+1}}-W_{t_{k}}\right)=\sum_{k=1}^{n-1}\left(t_{k+1}-t_{k}\right)=t_{n}-t_{1}
$$

which is in agreement with 1 . Consequently, 1 and 2 define joint distributions. In virtue of the Kolmogorov existence theorem there exists a random process $W^{*}=$ $=\left\{W_{t}^{*}, t \geqq 0\right\}, W_{0}^{*} \equiv 0$, complying with 1,2 . It is a Gaussian process with mean zero and covariance function, for $0 \leqq s \leqq t$,

$$
E W_{s}^{*} W_{t}^{*}=E\left(W_{t}^{*}-W_{s}^{*}\right) W_{s}^{*}+E\left(W_{s}^{*}\right)^{2}=s=\min (s, t) .
$$

Let us construct with the aid of $W^{*}$ a random process $W$ satisfying $1-3$. Define, for $n=1,2, \ldots,{ }^{n} W=\left\{{ }^{n} W_{t}, t \geqq 0\right\}$ by joining $W_{k 2-n}^{*}, k=0,1, \ldots$, by straight lines, i.e.

$$
\begin{gathered}
{ }^{n} W_{t}=2^{n}\left[\left(t-k 2^{-n}\right) W_{(k+1) 2-n}^{*}+\left((k+1) 2^{-n}-t\right) W_{k 2-n}^{*}\right], \\
k 2^{-n} \leqq t \leqq(k+1) 2^{-n} .
\end{gathered}
$$


Consider first the probability distribution of

$W_{t}^{*}-{ }^{n} W_{t}=2^{n}\left[\left((k+1) 2^{-n}-t\right)\left(W_{t}^{*}-W_{k 2-n}^{*}\right)-\left(t-k 2^{-n}\right)\left(W_{(k+1) 2^{-n}}^{*}-W_{t}^{*}\right)\right]$.

It is the normal distribution with mean 0 and variance

$$
E\left(W_{t}^{*}-{ }^{n} W_{t}\right)^{2}=2^{n}\left((k+1) 2^{-n}-t\right)\left(t-k 2^{-n}\right) \leqq 2^{-n-2} .
$$

The transition from ${ }^{n} W$ to ${ }^{n+1} W$ is the change of the straight lines joining $W_{k 2-n}^{*}$, $k=0,1, \ldots$, into triangles. Hence, for arbitrary positive integer $m$, and for $x>0$,

$$
\begin{gathered}
P\left(\left.\max _{i \in[0, m]}\right|^{n+1} W_{t}-{ }^{n} W_{t} \mid>x\right)=P\left(\max _{k=1, \ldots, m 2^{n}}\left|W_{(2 k-1) 2^{-n-1}-{ }^{n}}^{*} \mathrm{~W}_{(2 k-1) 2^{-n-1}}\right|>x\right) \leqq \\
\leqq \sum_{k=1}^{m 2^{n}} P\left(\left|W_{(2 k-1) 2-n-1}^{*}-{ }^{n} W_{(2 k-1) 2-n-1}\right|>x\right) \leqq \frac{m 2^{n+1}}{\sqrt{(2 \pi)}} \int_{x 2 n+2 / 2}^{\infty} \mathrm{e}^{-y^{2 / 2}} \mathrm{~d} y \leqq \\
\leqq \frac{m 2^{n+1}}{\sqrt{(2 \pi)}} \cdot \frac{1}{x 2^{n+2 / 2}} \mathrm{e}^{-x^{2} \cdot 2^{n+1}} .
\end{gathered}
$$

Setting $x_{n}=\sqrt{ }\left(n \log 2 / 2^{n}\right)$, one gets

$$
\sum_{n=1}^{\infty} P\left(\max _{t \in[0, m]}\left|{ }^{n+1} W_{t}-{ }^{n} W_{t}\right|>x_{n}\right)<\infty .
$$

From the Borel-Cantelli lemma follows that with probability 1

$$
\left|{ }^{n+1} W_{t}-{ }^{n} W_{t}\right| \leqq x_{n}, \quad t \in[0, m],
$$

for $n$ sufficiently large. $x_{n}, n=1,2, \ldots$, are terms of a convergent series. Thus, there exists a random process $W=\left\{W_{t}, t \geqq 0\right\}$ such that with probability 1

$$
\lim _{n \rightarrow \infty}{ }^{n} W_{t}=W_{t} \quad \text { uniformly in } \quad t \in[0, m],
$$

where $m>0$ is arbitrary. The uniform convergence implies that the trajectories of $W$ are almost surely continuous. From (11) by letting $n \rightarrow \infty$ one concludes that for $t \geqq 0, W_{t}=W_{t}^{*}$ a.s. Consequently, the joint distributions of $W$ and $W^{*}$ are the same. Hence, $W$ fulfils 1 and 2.

A Wiener process $W$ has rather irregular trajectories. One important property is stated in Lemma 1. 1.i.m. means limit in mean square.

Lemma 1. Let ${ }^{n} t_{0}=0<{ }^{n} t_{1}<\ldots<{ }^{n} t_{k_{n}}=T, n=1,2, \ldots$, be a sequence of divisions of interval $[0, T], T<\propto$. Denote

$$
{ }^{n} \Delta=\max _{j=c, \ldots, k_{n}-1}\left({ }^{n} t_{j+1}-{ }^{n} t_{j}\right), \quad{ }^{n} \xi=\sum_{j=0}^{k_{n}-1}\left(W_{n_{t_{j}+1}}-W_{n_{t_{j}}}\right)^{2} .
$$

10 
Then

$$
E^{n} \xi=T, \quad E\left({ }^{n} \xi-T\right)^{2}=E^{n} \xi^{2}-T^{2} \leqq 2 T^{n} \Delta, \quad n=1,2, \ldots
$$

If $\lim _{n \rightarrow \infty}{ }^{n} \Delta=0$, then l.i.m. $_{n \rightarrow \infty}{ }^{n} \xi=T$. If $\sum_{n=1}^{\infty}{ }^{n} \Delta<\infty$, then $\lim _{n \rightarrow \infty} n \xi=T$ a.s.

Proof.

$$
\begin{gathered}
E{ }^{n} \xi=\sum_{j} E\left(W_{n_{t_{j+1}}}-W_{n_{t_{j}}}\right)^{2}=\sum_{j}\left({ }^{n} t_{j+1}-{ }^{n} t_{j}\right)=T . \\
E(" \xi-T)^{2}=E\left(\sum_{j}\left[\left(W_{n_{t_{j+1}}}-W_{n_{t_{j}}}\right)^{2}-\left({ }^{n} t_{j+1}-{ }^{n} t_{j}\right)\right]\right)^{2}= \\
=\sum_{j} E\left[\left(W_{n_{t_{j+1}}}-W_{n_{t_{j}}}\right)^{2}-\left({ }^{n} t_{j+1}-{ }^{n} t_{j}\right)\right]^{2}=\sum_{j}\left[E\left(W_{n_{t_{j+1}}}-W_{n_{t_{j}}}\right)^{4}-\right. \\
\left.-\left({ }^{n} t_{j+1}-{ }^{n} t_{j}\right)^{2}\right]=2 \sum_{j}\left({ }^{n} t_{j+1}-{ }^{n} t_{j}\right)^{2} \leqq 2 T{ }^{n} \Delta .
\end{gathered}
$$

The next assertion of the lemma follows directly from (12). To establish the last one use the Borel-Cantelli lemma. For $\varepsilon>0$,

$$
\sum_{n=1}^{\infty} P\left(\left|{ }^{n} \xi-T\right| \geqq \varepsilon\right) \leqq \frac{1}{\varepsilon^{2}} \sum_{n=1}^{\infty} E\left({ }^{n} \xi-T\right)^{2} \leqq \frac{2 T}{\varepsilon^{2}} \sum_{n=1}^{\infty}{ }^{n} \Delta<\infty .
$$

Consequently, with probability $1,\left.\right|^{n} \xi-T \mid \geqq \varepsilon$ holds only for finitely many $n$.

The relation $\lim ^{n} \xi=T$ is stated in words by saying that the quadratic variation of $W$ on $[0, T]^{n \rightarrow \infty}$ equals $T$. The first order variation is infinite.

Corollary 1 . With probability 1 the trajectories of $W$ have unbounded variation on arbitrary interval $[S, T], 0 \leqq S<T<\infty$.

Proof. Let $S=0$, and use the denotations as above. It holds

$$
{ }^{n} \xi \leqq\left(\max _{i}\left|W_{n_{t_{i+1}}}-W_{n_{t_{i}}}\right|\right) \sum_{j}\left|W_{n_{t_{j+1}}}-W_{n_{t_{j}}}\right| .
$$

Assume $\sum_{n=1}^{\infty} n d<\infty$. The sum in (13) must be unbounded as $n \rightarrow \infty$, whenever $W_{t}$ is continuous on $[0, T]$, and, at the same time, " $\xi$ does not tend to 0 . But this occurs almost surely.

In virtue of Corollary 1, with probability 1 the trajectories of $W$ are not absolutely continuous, since absolute continuity implies boundedness of the variation on finite intervals. It can be shown that with probability 1 the trajectories are nowhere differentiable.

We call $r$-dimensional Wiener process a vector process $\bar{W}=\left\{\bar{W}_{t}=\left(W_{t}^{1}, \ldots, W_{t}^{r}\right)\right.$, $t \geqq 0\}$ the components of which are $r$ mutually independent Wiener processes. 


\subsection{Commentary}

The random process called nowadays the Wiener process (the Brownian motion process, the Wiener-Lévy process) was introduced by the Frenchman L. Bachelier (1900) as a model for the price fluctuations on a stock market. He defined it as the limit of a sequence of symmetric random walks. A. Einstein (1905) came upon the same process when developing the theory of the random movement of small particles in a fluid. This phenomenon was observed by the English botanist R. Brown (1826). The mathematical theory of the Brownian motion was initiated by N. Wiener (1923). $\mathrm{He}$ treated the probability distribution of the Brownian motion process as a measure in the space of continuous functions. The discovery of many important properties of the Wiener process is due to P. Lévy.

The Wiener process represents the integral of what is called in the applications the white noise. To get more insight into its nature, consider a theory of the Brownian movement, which extends Einstein's ideas. Let $X$ denote one coordinate of the Brownian particle. Its evolution is described by the following equation

$$
m \frac{\mathrm{d}^{2}}{\mathrm{~d} t^{2}} X_{t}+c \frac{\mathrm{d}}{\mathrm{d} t} X_{t}=F_{t}
$$

$m$ is the mass of the particle, $c$ is the coefficient of viscous friction. $F$ are the forces acting on the particle in the direction of the coordinate. (According to [2], for particles of colloidal platinum with the radius $2.5 \cdot 10^{-6} \mathrm{~cm}$ in water: $m=2.5$. $.10^{-15} \mathrm{~g}, c=7.5 \cdot 10^{-9} \mathrm{~g} / \mathrm{sec}$.) Take $t=0$ as the origin, and denote $(\mathrm{d} / \mathrm{d} t) X_{t}=\dot{X}_{t}$. From (14) one gets

$$
\begin{gathered}
\dot{X}_{t}=\dot{X}_{0} \mathrm{e}^{-(c / m) t}+\mathrm{e}^{-(c / m) t} \frac{1}{m} \int_{0}^{t} \mathrm{e}^{(c / m) s} F_{s} \mathrm{~d} s, \\
X_{t}=X_{0}+\int_{0}^{t} \dot{X}_{s} \mathrm{~d} s, \quad t \geqq 0 .
\end{gathered}
$$

Consider $F$. It results from the impacts on the particle of the molecules of the fluid in thermal motion. By the temperature of about $20^{\circ} \mathrm{C}$ the mean time between the collisions is of the order of $10^{-21}$ seconds. It is therefore difficult to imagine the trajectory of $F$. This is expressed by saying that $F$ has the character of the white noise, i.e. of a process the values of which are mutually independent random variables changing with an extremely high frequency. A more precise meaning can be given to the integral $\int_{0}^{t} F_{s} \mathrm{~d} s, t \geqq 0$. The following properties are intuitively plausible. 1. The increments $\int_{t_{1}}^{t_{2}} F_{s} \mathrm{~d} s$ have mean 0 . (The fluid is homogeneous, the shocks in positive or negative direction equiprobable.) Their distribution is the normal. (Sum of a large number of independent random quantities.) 2. The increments on non-overlapping time intervals are mutually independent, and their probability distribution 
is invariant with respect to the time shift. 3 . The integral is a continuous function of $t$. Thus, we arrived to the conclusion that

$$
\int_{0}^{t} F_{s} \mathrm{~d} s=\sigma W_{t}, \quad t \geqq 0 .
$$

$\sigma$ is a constant, whose value $\sqrt{ }(2 k T / c)$ is supplied by physical theories. The reader certainly noticed that Properties $1-3$ are not plausible for time intervals having length comparable to the mean time between the collisions.

Rewrite (15) using "Stieltjes integral",

$$
\dot{X}_{t}=\dot{X}_{0} \mathrm{e}^{-(c / m) t}+\mathrm{e}^{-(c / m) t} \frac{\sigma}{m} \int_{0}^{t} \mathrm{e}^{(c / m) s} \mathrm{~d} W_{s} .
$$

In the definition of the ordinary Stieltjes integral the weighting function is assumed to have bounded variation. This is with probability 1 not true in (16) by Corollary 1 . The difficulty is easily overcome in this case, since the integrand is nonrandom and continuously differentiable. Integrating by parts we get

$$
\int_{0}^{t} \mathrm{e}^{(c / m) s} \mathrm{~d} W_{s}=\mathrm{e}^{(c / m) t} W_{t}-\frac{c}{m} \int_{0}^{t} \mathrm{e}^{(c / m) s} W_{s} \mathrm{~d} s .
$$

Both terms on the right are well defined. However, ambiguities arise already, when we consider $\int_{0}^{t} W_{s} \mathrm{~d} W_{s}$.

Let us define $\int_{0}^{t} W_{s} \mathrm{~d} W_{s}$ as the limit of the sums

$$
\begin{aligned}
& I_{n}= \sum_{k=1}^{\left[t 2^{n}\right]} W_{(k-1) 2^{-n}}\left(W_{k 2-n}-W_{(k-1) 2-n}\right)+W_{\left[t 2^{n}\right] 2^{2-n}}\left(W_{t}-W_{\left[t 2^{n}\right]^{2-n}}\right)= \\
&=\frac{1}{2} \sum_{k=1}^{\left[t 2^{n}\right]}\left\{\left(W_{k 2-n}^{2}-W_{(k-1) 2^{-n}}^{2}\right)-\left(W_{k 2^{-n}}-W_{(k-1) 2-n}\right)^{2}\right\}+ \\
& \quad+\frac{1}{2}\left\{\left(W_{t}^{2}-W_{\left[t 2^{n}\right] 2^{-n}}^{2}\right)-\left(W_{t}-W_{\left[t 2^{n}\right] 2-n}\right)^{2}\right\} .
\end{aligned}
$$

[a] denotes the integer part of $a$. From here using Lemma 1 one gets

$$
\int_{0}^{t} W_{s} \mathrm{~d} W_{s}=\lim _{n \rightarrow \infty} I_{n}=\frac{1}{2} W_{t}^{2}-\frac{1}{2} t \quad \text { a.s., } \quad t \geqq 0 .
$$

Notice the disagreement of (17) with the rules of ordinary calculus.

If we change the ordinates of the integrand from $W_{(k-1) 2^{-n}}$ to $W_{k 2^{-n}}$, we get a result different from (17). Set

$$
\begin{aligned}
J_{n} & =\sum_{k=1}^{\left[t 2^{n}\right]} W_{k 2^{-n}}\left(W_{k 2^{-n}}-W_{(k-1) 2^{-n}}\right)+W_{t}\left(W_{t}-W_{\left[t 2^{n}\right] 2-n}\right)= \\
& =I_{n}+\sum_{k=1}^{\left[t 2^{n}\right]}\left(W_{k 2^{-n}}-W_{(k-1) 2-n}\right)^{2}+\left(W_{t}-W_{\left[2^{n}\right]^{n-n}}\right)^{2} .
\end{aligned}
$$


Hence,

$$
\lim _{n \rightarrow \infty} J_{n}=\frac{1}{2} W_{t}^{2}+\frac{1}{2} t \quad \text { a.s., } \quad t \geqq 0 .
$$

Definition is a matter of practicality. Let us mention that the appropriate definition of $\int_{0}^{t} W_{s} \mathrm{~d} W_{s}$ is the former one giving the stochastic integral in Itô's sense. It can be generalized, and the integral has good properties. Essential is the fact that $W_{(k-1) 2-n}$. . $\left(W_{k 2-n}-W_{(k-1) 2-n}\right)$ are products of independent random variables. Itô's definition of the stochastic integral leads to a differential calculus not conformal to the ordinary one. Namely, (17) can be written as

$$
W_{t}^{2}=2 \int_{0}^{t} W_{s} \mathrm{~d} W_{s}+t, \quad t \geqq 0 .
$$

Hence, passing to the differentials,

$$
\mathrm{d}\left(W_{t}^{2}\right)=2 W_{t} \mathrm{~d} W_{t}+\mathrm{d} t
$$

The principal rule for dealing with stochastic differentials is the Ito formula. We remark that

$$
\lim _{n \rightarrow \infty} \frac{1}{2}\left(I_{n}+J_{n}\right)=\frac{1}{2} W_{t}^{2} \text { a.s., } t \geqq 0,
$$

in accordance with the ordinary calculus. But this advantage of the stochastic integral in Stratonovich's sense is overshadowed by its considerably worse mathematical tractability.

(14) is a stochastic differential equation, the Langevin equation. The introduction of differentials and the substitution $c / m=a, \sigma / m=b$ transforms (14) into

$$
\mathrm{d} \dot{X}_{t}=-a \dot{X}_{t} \mathrm{~d} t+b \mathrm{~d} W_{t}, \quad t \geqq 0 .
$$

From (18), it follows

$$
\begin{gathered}
\dot{X}_{t+h}=\dot{X}_{t} \mathrm{e}^{-a h}+b \int_{0}^{h} \mathrm{e}^{-a(h-s)} \mathrm{d} W_{s+t}=\dot{X}_{t} \mathrm{e}^{-a h}+ \\
+b\left(W_{t+h}-W_{t}\right)+a b \int_{0}^{h} \mathrm{e}^{-a(h-s)}\left(W_{t+s}-W_{t}\right) \mathrm{d} s, \quad h>0, \quad t \geqq 0 .
\end{gathered}
$$

Since the increments of $W$ are independent random variables, the last two terms are independent of

$$
\dot{X}_{u}=\dot{X}_{0} \mathrm{e}^{-a u}+b \int_{0}^{u} \mathrm{e}^{-a(u-s)} \mathrm{d} W_{s}, \quad u \in[0, t],
$$

provided that $\dot{X}_{0}$ is independent of $W$. Consequently, $\dot{X}_{t+h}$ depends on $\left\{\dot{X}_{u}, u \in[0, t]\right\}$ only through $\dot{X}_{t} . \dot{X}$ is therefore a Markov process. 


\section{INCREASING FAMILIES OF $\sigma$-ALGEBRAS}

\subsection{Definitions}

Stochastic analysis is in the first place a tool for studying the dynamics of random phenomena or the random evolution in time. It is therefore necessary to perform a time structuring of random events. Mostly, this structuring is inherent to the physical interpretation of the events. Let on $(\Omega, \mathscr{A}, P)$ be defined $n$ random processes ${ }^{1} X=$ $=\left\{{ }^{1} X_{t}, t \geqq 0\right\}, \ldots,{ }^{n} X=\left\{{ }^{n} X_{t}, t \geqq 0\right\}$. Consider a possibly fictive observer of ${ }^{1} X, \ldots,{ }^{n} X$. The random events about which he can tell at time $t$, whether they occurred or not constitute the $\sigma$-algebra

$$
\begin{gathered}
\mathscr{F}_{t}=\sigma a\left({ }^{1} X_{s}, s \in[0, t], \ldots,{ }^{n} X_{s}, \quad s \in[0, t]\right)= \\
=\sigma a\left(\left\{{ }^{i} X_{s} \leqq x\right\}, \quad s \in[0, t], \quad x \in(-\infty, \infty), \quad i=1, \ldots, n\right) .
\end{gathered}
$$

$\sigma a$ ( ) denotes the $\sigma$-algebra generated by the random events (random processes) in the brackets. For $0 \leqq t_{1}<t_{2}, \mathscr{F}_{t_{1}} \subset \mathscr{F}_{t_{2}} . \mathscr{F}=\left\{\mathscr{F}_{t}, t \geqq 0\right\}$ describes the growth of the observer's information in time.

Any increasing (precisely nondecreasing) family of $\sigma$-algebras $\mathscr{F}=\left\{\mathscr{F}_{t}, t \geqq 0\right\}$, $\mathscr{F}_{t} \subset \mathscr{A}$, defines the time dynamics of $\mathscr{A}$. We interpret $\mathscr{F}_{t}$ as the collection of random events, for which at time $t$ is given - at the level of observability appropriate to the problem under consideration -- whether they took place or not. We shall in the sequel always assume that the basic space $(\Omega, \mathscr{A}, P)$ is equipped with an increasing family of $\sigma$-algebras $\mathscr{F}=\left\{\mathscr{F}_{t}, t \geqq 0\right\}$. The random events from $\mathscr{F}_{t}$ can be briefly called events prior to $t$.

Next we consider random functions $Y=\left\{Y_{t}, t \geqq 0\right\}$ having the property that for $t \geqq 0$ the value of $Y_{t}$ is fixed not later than at time $t$. The property is named nonanticipativity. If $\mathscr{F}$ is defined as in (1), then the values taken by

$$
Y_{t}=f\left({ }^{1} X_{t}, \ldots,{ }^{n} X_{t}\right), \quad Y_{t}=\int_{0}^{t} f\left({ }^{1} X_{s}, \ldots,{ }^{n} X_{s}\right) \mathrm{d} s,
$$

where $f$ is a Borel function, are known to the observer at time $t$. Formally we define nonanticipativity as progressive measurability with respect to $\mathscr{F}$.

Definition 1. Random process $Y=\left\{Y_{t}, t \geqq 0\right\}$ is called nonanticipative, if, for $t \geqq 0,\left\{Y_{s}, s \in[0, t]\right\}$ is a measurable random process on $\left(\Omega, \mathscr{F}_{t}, P\right)$.

Recall our convention that a random process is a measurable process. Definition 1 enables us to use the Fubini theorem with regard to $\mathscr{B}_{[0, t]} \times \mathscr{F}_{t}$. For example, if $Y$ is nonanticipative, then $\int_{0}^{t} f\left(Y_{s}\right) \mathrm{d} s$ is $\mathscr{F}_{t}$-measurable provided that the integral exists for all trajectories. The property that $Y_{t}$ is $\mathscr{F}_{t}$-measurable for $t \geqq 0$ will be stated as $Y$ complies with $\mathscr{F}$. Theorem 1.2 has the following obvious consequence. 
Theorem 1. If $Y$ complies with $\mathscr{F}$ and has right-continuous (left-continuous) trajectories, then it is nonanticipative.

Return now to the Wiener process, and let us reformulate its property of independence of the increments. The trajectories of $W=\left\{W_{t}, t \geqq 0\right\}$ induce the family

$$
\mathscr{F}^{W}=\left\{\mathscr{F}_{t}^{W}=\sigma a\left(W_{s}, s \in[0, t]\right), \quad t \geqq 0\right\} .
$$

We shall verify that 2 of Definition 1.3 implies for $t \geqq 0, s>0$, the independence of $W_{t+s}-W_{t}$ and $\widetilde{F}_{t}^{W}$. The events

$$
\begin{gathered}
\left\{\left(W_{t_{1}}, W_{t_{2}}-W_{t_{1}}, \ldots, W_{t_{n}}-W_{t_{n-1}}\right) \in A\right\}, t_{1}, \ldots, t_{n} \in[0, t], \\
A \quad \text { Borel set in } R^{n}, \quad n=1,2, \ldots,
\end{gathered}
$$

form an algebra which generates $\mathscr{F}_{t}^{W}$. We have to demonstrate

$$
P\left(\left\{W_{t+s}-W_{t} \in B\right\} \cap M\right) . P\left(W_{t+s}-W_{t} \in B\right)^{-1}=P(M), \quad M \in \mathscr{F}_{t}^{W} .
$$

$B$ is a Borel set, $P\left(W_{t+s}-W_{t} \in B\right)>0$. For fixed $B$ the conditional probability on the right-hand side of (3) agrees with $P$ for $M$ of the form (2), in virtue of 2 of Definition 1.3. Thus, (3) is a consequence of the unicity of the extension of $P$ to the least $\sigma$-algebra $\mathscr{F}_{t}^{W}$.

It is convenient to replace $\mathscr{F}^{W}$ by the basic family $\mathscr{F}$. This leads to the next definition.

Definition 2. Random process $W=\left\{W_{t}, t \geqq 0\right\}$ on $(\Omega, \mathscr{A}, P)$ is a Wiener process with respect to increasing family of $\sigma$-algebras $\mathscr{F}=\left\{\mathscr{F}_{t}, t \geqq 0\right\}$, if the following holds.

1. For $t \geqq 0, s>0$, the increments $W_{t+s}-W_{t}$ are distributed normally with mean 0 and variance $s$.

2. $W$ complies with $\mathscr{F}$, and for $t \geqq 0, s>0, W_{t+s}-W_{t}$ is independent of $\mathscr{F}_{t}$.

3. The trajectories of $W$ are continuous almost surely.

The multidimensional version of Definition 2 is straightforward.

If $W$ satisfies Definition 2, then

$$
0=E\left\{W_{t}-W_{s} \mid \mathscr{F}_{s}\right\} \quad \text { or } \quad E\left\{W_{t} \mid \mathscr{F}_{s}\right\}=W_{s}, \quad 0 \leqq s \leqq t .
$$

This property characterizes a martingale.

\subsection{Martingales}

In terms of the games of chance a martingale describes a succession of fair games. In such games the player stakes an amount equal to his expected gain. The founder of the mathematical theory of martingales is J. L. Doob (1953), who continued the investigations of P. Lévy (1937) and of J. Ville (1939). 
Definition 3. Random process $X=\left\{X_{t}, t \in J\right\}$ is a martingale, with respect to increasing family of $\sigma$-algebras $\mathscr{F}=\left\{\mathscr{F}_{t}, t \in J\right\}$, if $E\left|X_{t}\right|<\infty, t \in J$, and

$$
E\left\{X_{t} \mid \mathscr{F}_{s}\right\}=X_{s}, \quad s \leqq t, \quad s, t \in J .
$$

Example 1. Let $W$ be as in Definition 2, and let $a$ be a real number. Set

$$
Y_{t}=\exp \left\{a W_{t}-\frac{1}{2} a^{2} t\right\}, \quad t \geqq 0 .
$$

Then

$$
\begin{gathered}
E\left\{Y_{t} \mid \mathscr{F}_{s}\right\}=E\left\{\exp \left\{a\left(W_{t}-W_{s}\right)-\frac{1}{2} a^{2}(t-s)\right\} \mid \mathscr{F}_{s}\right\} Y_{s}=Y_{s}, \\
0 \leqq s \leqq t .
\end{gathered}
$$

Thus $Y$ is a martingale with respect to $\mathscr{F}$.

Any martingale $X$ is a martingale with respect to the family $\mathscr{F}^{X}=\left\{\sigma a\left(X_{s}, s \leqq t\right)\right.$, $t \in J\}$. If $X$ satisfies Definition 3, then by Property 1.3 the process $Y_{t}=\left|X_{t}\right|, t \in J$, satisfies

$$
E\left\{Y_{t} \mid \mathscr{F}_{s}\right\} \geqq Y_{s}, \quad s, t, \quad s, t \in J .
$$

The same holds for $Y_{t}=X_{t}^{2}, t \in J$, provided $E X_{t}^{2}<\infty, t \in J$, in virtue of Property 1.6 .

Random process $Y=\left\{Y_{t}, t \in J\right\}$ complying with $\mathscr{F}$, and such that $E\left|Y_{t}\right|<\infty$, $t \in J$, and (4) hold is a submartingale with respect to $\mathscr{F}$. Submartingales correspond to successions of fair or favorable games.

Theorem 2. (Doob's submartingale inequality.) Let $Y=\left\{Y_{t}, t \in[0, T]\right\}, T<\infty$, be a submartingale with right-continuous (or left-continuous) trajectories. Then

$$
P\left(\sup _{t \in[0, T]} Y_{t} \geqq x\right) \leqq x^{-1} E Y_{T}^{+}, \quad x>0,
$$

where $Y_{T}^{+}=\max \left(Y_{T}, 0\right)$.

Proof. Set $Z_{k}=Y_{k T / n}, k=0, \ldots, n$. First we show that

$$
P\left(\sup _{k=0, \ldots, n} Z_{k} \geqq x\right)=x^{-1} E Z_{n}^{+} .
$$

Denote the random event in brackets by $B$. It is the union of disjoint events

$$
B_{k}=\bigcap_{j<k}\left\{Z_{j}<x\right\} \cap\left\{Z_{k} \geqq x\right\}, k=0, \ldots, n .
$$

It holds

$$
\begin{gathered}
\left\{Z_{j}<x\right\}=\left\{Y_{j T / n}^{\prime}<x\right\} \in \mathscr{F}_{j T / n}^{Y} \subset \mathscr{F}_{k T / n}^{Y}, j<k, \\
\left\{Z_{k} \geqq x\right\} \in \mathscr{F}_{k T / n}^{Y} .
\end{gathered}
$$


Hence, $B_{k} \in \mathscr{F}_{k T / n}^{Y}$, and consequently,

$$
\begin{gathered}
E Z_{n}^{+} \geqq E \chi_{B} Z_{n}^{+} \geqq E \chi_{B} Z_{n}=\sum_{k=0}^{n} E \chi_{B_{k}} Z_{n}=\sum_{k=0}^{n} E E\left\{\chi_{B_{k}} Z_{n} \mid \mathscr{F}_{k T / n}^{Y}\right\}= \\
=\sum_{k=0}^{n} E \chi_{B_{k}} E\left\{Z_{n} \mid \mathscr{F}_{k T / n}^{Y}\right\} \geqq \sum_{k=0}^{n} E \chi_{B_{k}} Z_{k} \geqq x \sum_{k=0}^{n} P\left(B_{k}\right)=x P(B) .
\end{gathered}
$$

Thus, (6) is proved.

Letting $n \rightarrow \infty$, we get from (6)

$$
P\left(\sup _{t \in[0, T]} Y_{t}>x\right) \leqq x^{-1} E Y_{T}^{+} .
$$

To obtain (5), we write the above inequality for $x^{\prime}<x$, and let $x^{\prime} \rightarrow x$.

\subsection{Stopping Times}

Let $X=\left\{X_{t}, t \geqq 0\right\}$ be a continuous random process, $I$ an open interval of real numbers, and $x$ a real number. Random times

$$
\tau=\inf \left\{t: X_{t} \notin I\right\}, \quad \tau^{\prime}=\inf \left\{t: \int_{0}^{t}\left|X_{s}\right| \mathrm{d} s \geqq x\right\},
$$

have the property of being determined by $X_{t}, t \in[0, \tau]$ and $t \in\left[0, \tau^{\prime}\right]$, respectively. This is, for example, not true about $\tau^{\prime \prime}=\frac{1}{2} \tau$. The mentioned property can be stated as

$$
\begin{gathered}
\{\tau \leqq t\}=\left\{\sup _{s \in[0, t]} X_{s} \notin I \text { or } \inf _{s \in[0, t]} X_{s} \notin I\right\} \in \mathscr{F}_{t}^{X}=\sigma a\left(X_{s}, s \in[0, t]\right), \\
\left\{\tau^{\prime} \leqq t\right\}=\left\{\int_{0}^{t}\left|X_{s}\right| \mathrm{d} s \geqq x\right\} \in \mathscr{F}_{t}^{X}, \quad t \geqq 0 .
\end{gathered}
$$

Definition 4. Nonnegative (possibly infinite) random variable $\tau$ is a stopping time with respect to family $\mathscr{F}=\left\{\mathscr{F}_{t}, t \geqq 0\right\}$ if $\{\tau \leqq t\} \in \mathscr{F}_{t}, t \geqq 0$.

The independence of $\tau$ on the future is expressed in the statement that at any time $t$ it is given, whether $\tau \leqq t$ or not. Stopping times are also called Markovian times. Under additional hypotheses, the relations characterizing Markov processes or martingales can be extended from nonrandom times to stopping times.

Example 2. Let $W=\left\{W_{t}, t \geqq 0\right\}, W_{0} \equiv 0$, be a Wiener process, $a>0, \tau=$ inf. . $\left\{t: W_{t}=a\right\}$. Reflecting the trajectory around the straight line parallel to the time axis at distance $a$, one gets

$$
\begin{gathered}
P(\tau \leqq t)=P\left(\sup _{s \in[0, t]} W_{s} \geqq a\right)=P\left(W_{t} \geqq a\right)+ \\
+P\left(\sup _{s \in[0, t]} W_{s} \geqq a ; W_{t}<a\right)=2 P\left(W_{t} \geqq a\right)=\sqrt{ }(2 / \pi) \int_{a / \sqrt{ } t}^{\infty} \mathrm{e}^{-\left(x^{2} / 2\right)} \mathrm{d} x .
\end{gathered}
$$




\section{STOCHASTIC INTEGRAL}

\subsection{Simple Functions}

We assume a basic space $(\Omega, \mathscr{A}, P)$ with an increasing family of $\sigma$-algebras $\mathscr{F}=$ $=\left\{\mathscr{F}_{t}, t \geqq 0\right\}$. Let $\mathscr{F}_{0}$ comprise all sets of $P$-measure 0 , and let $W=\left\{W_{t}, t \geqq 0\right\}$, $W_{0} \equiv 0$, be a Wiener process on $(\Omega, \mathscr{A}, P)$. Everywhere in the text, without saying it, we mean Wiener process with respect to $\mathscr{F}$. Similarly, nonanticipavity, martingale property, and property of being a stopping time refer to $\mathscr{F}$, unless stated otherwise. We are going to define $\int_{0}^{t} \Phi_{s} \mathrm{~d} W_{s}$ for a sufficiently broad class of nonanticipative random functions $\Phi=\left\{\Phi_{t}, t \in[0, T]\right\}$.

To begin with, consider the approach using partial integration already mentioned in $\S 1$. Let $f$ be a nonrandom function on $[0,1]$ having continuous derivative $f^{\prime}$. Write

$$
\int_{0}^{1} f(s) \mathrm{d} W_{s}=f(1) W_{1}-\int_{0}^{1} f^{\prime}(s) W_{s} \mathrm{~d} s
$$

The right-hand side makes sense, and can be taken as definition of the integral. Further,

$$
\begin{gathered}
E \int_{0}^{1} f(s) \mathrm{d} W_{s}=f(1) E W_{1}-\int_{0}^{1} f^{\prime}(s) E W_{s} \mathrm{~d} s=0 . \\
E\left(\int_{0}^{1} f(s) \mathrm{d} W_{s}\right)^{2}=f(1)^{2} E W_{1}^{2}+\int_{0}^{1} \int_{0}^{1} f^{\prime}(t) f^{\prime}(s) E W_{t} W_{s} \mathrm{~d} t \mathrm{~d} s- \\
-2 f(1) \int_{0}^{1} f^{\prime}(s) E W_{1} W_{s} \mathrm{~d} s=f(1)^{2}+2 \int_{0}^{1} \int_{0}^{s} t f^{\prime}(t) \mathrm{d} t f^{\prime}(s) \mathrm{d} s- \\
-2 f(1) \int_{0}^{1} s f^{\prime}(s) \mathrm{d} s=f(1)^{2}-2 \int_{0}^{1} s f^{\prime}(s) f(s) \mathrm{d} s=\int_{0}^{1} f(s)^{2} \mathrm{~d} s .
\end{gathered}
$$

Denote by $L^{2}[0,1]$ the Hilbert space of quadratically integrable functions on $[0,1]$. (1) defines an isometric mapping of a dense subset of $L^{2}[0,1]$ onto a subset of the Hilbert space of random variables on $(\Omega, \mathscr{A}, P)$ with finite second moment. The mapping can be extended on the entire $L^{2}[0,1]$. This gives $\int_{0}^{1} f(s) \mathrm{d} W_{s}$ for $f \in L^{2}[0,1]$. A similar isometry can be used to define integrals of nonanticipative functions. One has to assume

$$
\int_{0}^{1} E \Phi_{s}^{2} \mathrm{~d} s<\infty .
$$

We shall define the integral under a weaker assumption than (2), and introduce the isometry later on.

Take an arbitrary interval $[0, T], 0<T<\infty$. 
Definition 1. Random function $\Phi=\left\{\Phi_{t}, t \in[0, T]\right\}$ is a simple function, if it is nonanticipative, and if there exists a division $t_{0}=0<t_{1}<t_{2}<\ldots<t_{n}=T$ of interval $[0, T]$ together with random variables $\varphi_{0}, \ldots, \varphi_{n-1}$ such that

$$
\Phi_{t}=\varphi_{j}, \quad t_{j} \leqq t<t_{j+1}, \quad j=0, \ldots, n-1 .
$$

The nonanticipativity assumption implies that $\varphi_{j}$ is $\mathscr{F}_{i j}$-measurable, $j=0, \ldots$ $\ldots, n-1$.

Definition 2. Let $\Phi$ be a simple function as in Definition 1. For $t \in[0, T]$ we define its stochastic integral as

$$
\int_{0}^{t} \Phi_{s} \mathrm{~d} W_{s}=\sum_{j=0}^{k-1} \varphi_{j}\left(W_{t_{j+s}}-W_{t_{j}}\right)+\varphi_{k}\left(W_{t}-W_{t_{k}}\right) \text { if } t_{k} \leqq t \leqq t_{k+1} .
$$

(For $k=0$ the sum on the right-hand side is zero.)

The following properties of integrals of simple functions are obvious:

1. $\int_{0}^{t} \Phi \mathrm{d} W$ is the same for all divisions of $[0, T]$ such that (3) holds.

2. $\int_{0}^{t}\left(a \Phi+a^{t} \Phi^{\prime}\right) \mathrm{d} W=a \int_{0}^{t} \Phi \mathrm{d} W+a^{\prime} \int_{0}^{t} \Phi^{\prime} \mathrm{d} W, \quad t \in[0, T]$, for arbitrary constants $a, a^{\prime}$.

3. $\int_{0}^{t} \Phi \mathrm{d} W$ is a continuous nonanticipative (random) function of $t$ on $[0, T]$.

Random process $Z$ introduced bellow has numerous applications in stochastic analysis. Next it will be used to derive an inequality for stochastic integrals.

Lemma 1. Random process

$$
Z_{t}=\exp \left\{\int_{0}^{t} \Phi_{s} \mathrm{~d} W_{s}-\frac{1}{2} \int_{0}^{t} \Phi_{s}^{2}\right\} \mathrm{d} s, \quad t \in[0, T],
$$

is a martingale with respect to $\mathscr{F}$, and $E Z_{T}=1$.

Proof. The nonanticipativity of $Z$ is easily seen. Further, take arbitrary $s \in[0, T)$. Let (3) hold, and let $t_{k} \leqq s<t_{k+1}$. It suffices to verify the martingale property for $t$ satisfying $s<t \leqq t_{k+1}$. The verification for $t_{k+1}<t \leqq t_{k+2}$ etc. follows then from the equalities

$$
E\left\{Z_{t} \mid \mathscr{F}_{s}\right\}=E\left\{E\left\{Z_{t} \mid \mathscr{F}_{t_{k+1}}\right\} \mid \mathscr{F}_{s}\right\}=E\left\{Z_{t_{k+1}} \mid \mathscr{F}_{s}\right\}=Z_{s} .
$$

We have for $s<t \leqq t_{k+1}$

$$
E\left\{Z_{t} \mid \mathscr{F}_{s}\right\}=Z_{s} E\left\{\exp \left\{\varphi_{k}\left(W_{t}-W_{s}\right)-\frac{1}{2} \varphi_{k}^{2}(t-s)\right\} \mid \mathscr{F}_{s}\right\}=Z_{s},
$$

since $\varphi_{k}$ is $\mathscr{F}_{s}$-measurable, and the conditional distribution of $\varphi_{k}\left(W_{t}-W_{s}\right)$ is normal with mean 0 and variance $\varphi_{k}^{2}(t-s)$. Finally,

$$
E Z_{T}=E E\left\{Z_{T} \mid \mathscr{F}_{t_{n-1}}\right\}=E Z_{t_{n-1}}=\ldots=E Z_{0}=1 .
$$


Lemma 2. For arbitrary positive numbers $a, b$,

(4) $\quad P\left(\sup _{t \in[0, T]}\left|\int_{0}^{t} \Phi \mathrm{d} W\right| \geqq a\right) \leqq P\left(\int_{0}^{T} \Phi^{2} \mathrm{~d} s \geqq b\right)+2 \exp \left\{-\frac{a^{2}}{2 b}\right\}$.

Proof. Apply Doob's inequality (Theorem 2.2) to martingale

It follows

$$
Z_{t}=\exp \left\{\frac{a}{b} \int_{0}^{t} \Phi \mathrm{d} W-\frac{1}{2}\left(\frac{a}{b}\right)^{2} \int_{0}^{t} \Phi^{2} \mathrm{~d} s\right\}, \quad t \in[0, T]
$$

$$
\begin{gathered}
P\left(\sup _{t \in[0, T]}\left(\int_{0}^{t} \Phi \mathrm{d} W-\frac{a}{2 b} \int_{0}^{t} \Phi^{2} \mathrm{~d} s\right) \geqq \frac{a}{2}\right)=P\left(\sup _{t \in[0, T]} Z_{t} \geqq\right. \\
\left.\geqq \exp \frac{a^{2}}{2 b}\right) \leqq \exp \left\{-\frac{a^{2}}{2 b}\right\} E Z_{T}=\exp \left\{-\frac{a^{2}}{2 b}\right\}
\end{gathered}
$$

From here and from the same inequality for $-\Phi$ one gets

$$
P\left(\sup _{t \in[0, T]}\left|\int_{0}^{t} \Phi \mathrm{d} W\right| \geqq \frac{a}{2}+\frac{a}{2 b} \int_{0}^{T} \Phi^{2} \mathrm{~d} s\right) \leqq 2 \exp \left\{-\frac{a^{2}}{2 b}\right\} .
$$

Further,

$$
\begin{gathered}
\left\{\sup _{t \in[0, T]}\left|\int_{0}^{t} \Phi \mathrm{d} W\right| \geqq a\right\} \subset\left\{\int_{0}^{T} \Phi^{2} \mathrm{~d} s \geqq b\right\} \cup\left\{\sup _{t \in[0, T]}\left|\int_{0}^{t} \Phi \mathrm{d} W\right| \geqq\right. \\
\left.\geqq \frac{a}{2}+\frac{a}{2 b} \int_{0}^{T} \Phi^{2} \mathrm{~d} s\right\} .
\end{gathered}
$$

This together with (5) implies (4).

We conclude this section by computing the variance of $\int_{0}^{t} \Phi \mathrm{d} W$.

Lemma 3. Let $\Phi$ be as in Definition 1, and let $E \varphi_{j}^{2}<\infty, j=0, \ldots, n-1$. Then

$$
E\left(\int_{0}^{t} \Phi \mathrm{d} W\right)^{2}=E \int_{0}^{t} \Phi^{2} \mathrm{~d} s, \quad t \in[0, T] .
$$

Proof. If $t_{k} \leqq t \leqq t_{k+1}$, then

$$
\begin{aligned}
& E\left(\int_{0}^{t} \Phi \mathrm{d} W\right)^{2}=\sum_{j=0}^{k-1} E \varphi_{j}^{2} E\left(W_{t_{j+1}}-W_{t_{j}}\right)^{2}+E \varphi_{k}^{2} E\left(W_{i}-W_{t_{k}}\right)^{2}= \\
& =\sum_{j=0}^{k-1} E \varphi_{j}^{2}\left(t_{j+1}-t_{j}\right)+E \varphi_{k}^{2}\left(t-t_{k}\right)=\int_{0}^{t} E \Phi^{2} \mathrm{~d} s=E \int_{0}^{t} \Phi^{2} \mathrm{~d} s,
\end{aligned}
$$

since, for $j=1, \ldots, k$, is $\varphi_{j}$ independent of $W_{t_{i+1}}-W_{t_{i}}, j \leqq i<k$, and of $W_{t}-W_{t_{k}}$. 


\subsection{Definition}

Stochastic integral will be defined for nonanticipative functions $\Phi=\left\{\Phi_{t}, t \in\right.$ $\in[0, T]\}$ satisfying

$$
\int_{0}^{T} \Phi_{s}^{2} \mathrm{~d} s<\infty \text { a.s. }
$$

To its extension from simple functions the property of the convergence in probability will be used that a Cauchy sequence has a limit. This is well known for random variables, and obviously valid also for continuous random processes.

Lemma 4. Let ${ }^{n} X=\left\{{ }^{n} X_{t}, t \in[0, T]\right\}, n=1,2, \ldots$, be a sequence of random processes with continuous trajectories, and such that for each $\varepsilon>0$

$$
\lim _{\substack{m \rightarrow \infty \\ n \rightarrow \infty}} P\left(\sup _{t \in[0, T]}\left|{ }^{m} X_{t}-{ }^{n} X_{t}\right| \geqq \varepsilon\right)=0 .
$$

Then there exists a continuous random process $X=\left\{X_{t}, t \in[0, T]\right\}$ such that for each $\varepsilon>0$

$$
\lim _{n \rightarrow \infty} P\left(\sup _{t \in[0, T]}\left|{ }^{n} X_{t}-X_{t}\right| \geqq \varepsilon\right)=0 .
$$

We remind the convention about indistinguishability made in $\S 1.1$.

Nonanticipative functions, satisfying (7), can be approximated by simple functions in the following sense.

Lemma 5. Let $\Phi$ be a nonanticipative function, and let (7) hold. Then there exists a sequence of simple functions $\left\{{ }^{n} \Phi, n=1,2, \ldots\right\}$ for which

$$
\mathrm{p} \lim _{n \rightarrow \infty} \int_{0}^{T}\left({ }^{n} \Phi_{s}-\Phi_{s}\right)^{2} \mathrm{~d} s=0 .
$$

( $\mathrm{p}$ lim stands for limit in probability.)

Proof. If $\Phi$ is continuous, then (8) holds for

$$
{ }^{n} \Phi_{t}=\Phi_{[t n] / n}, \quad t \in[0, T], \quad n=1,2, \ldots,
$$

where $[a]$ in the subscript denotes the integer part of $a$. In the general case, $\Phi$ is approximated first by continuous functions

$$
{ }^{h} \Phi_{t}=\frac{1}{h} \int_{t-h}^{t} \Phi_{s} \mathrm{~d} s, \quad t \in[0, T], \quad h>0 .
$$


In (9) we let $\Phi_{s}=0$ for $s \notin[0, T]$. By a theorem on quadratically integrable functions, (7) implies

$$
\lim _{h \rightarrow 0} \int_{0}^{T}\left({ }^{h} \Phi_{s}-\Phi_{s}\right)^{2} \mathrm{~d} s=0 \text { a.s. }
$$

Let $\Phi$ be a nonanticipative function satisfying (7). Take a sequence of simple functions $\left\{{ }^{n} \Phi, n=1,2, \ldots\right\}$ such that (8) holds. Then

$$
\mathrm{p} \lim _{\substack{m \rightarrow \infty \\ n \rightarrow \infty}} \int_{0}^{T}\left({ }^{m} \Phi_{s}-{ }^{n} \Phi_{s}\right)^{2} \mathrm{~d} s=0 .
$$

This is a consequence of $(8)$ and of the inequality

$$
\left(\int_{0}^{T}\left({ }^{m} \Phi_{s}-{ }^{n} \Phi_{s}\right)^{2} \mathrm{~d} s\right)^{1 / 2} \leqq\left(\int_{0}^{T}\left({ }^{m} \Phi_{s}-\Phi_{s}\right)^{2} \mathrm{~d} s\right)^{1 / 2}+\left(\int_{0}^{T}\left({ }^{n} \Phi_{s}-\Phi_{s}\right)^{2} \mathrm{~d} s\right)^{1 / 2} .
$$

Let us show that the random processes $\left\{\int_{0}^{t}{ }^{n} \Phi \mathrm{d} W, t \in[0, T]\right\}, n=1,2, \ldots$, form a Cauchy sequence in probability, i.e.

$$
\lim _{\substack{m \rightarrow \infty \\ n \rightarrow \infty}} P\left(\sup _{t \in[0, T]}\left|\int_{0}^{t}\left({ }^{m} \Phi-{ }^{n} \Phi\right) \mathrm{d} W\right| \geqq \varepsilon\right)=0 \text { for } \varepsilon>0 .
$$

By Lemma 2, for $b>0$,

$$
\begin{gathered}
\varlimsup_{\substack{m \rightarrow \infty \\
n \rightarrow \infty}} P\left(\sup _{t \in[0, T]}\left|\int_{0}^{t}\left({ }^{m} \Phi-{ }^{n} \Phi\right) \mathrm{d} W\right| \geqq \varepsilon\right) \leqq \varlimsup_{\substack{m \rightarrow \infty \\
n \rightarrow \infty}} P\left(\int_{0}^{T}\left({ }^{m} \Phi-{ }^{n} \Phi\right)^{2} \mathrm{~d} s \geqq b\right)+ \\
+2 \exp \left\{-\frac{\varepsilon^{2}}{2 b}\right\}=2 \exp \left\{-\frac{\varepsilon^{2}}{2 b}\right\}
\end{gathered}
$$

From here (11) is obtained by letting $b \rightarrow 0$.

In virtue of (11) there exists, according to Lemma 4 , a continuous random process $I=\left\{I_{t}, t \in[0, T]\right\}$ such that

$$
\lim _{n \rightarrow \infty} P\left(\sup _{t \in[0, T]}\left|\int_{0}^{t} \Phi^{n} \mathrm{~d} W-I_{t}\right| \geqq \varepsilon\right)=0 \text { for } \varepsilon>0 .
$$

Definition 3. We define

$$
\int_{0}^{t} \Phi_{s} \mathrm{~d} W_{s}=I_{t}, \quad t \in[0, T]
$$

Up to indistinguishability the definition is unique. It does not depend on the chosen sequence $\left\{{ }^{n} \Phi, n=1,2, \ldots\right\}$ of simple functions. To show it, consider 
another sequence $\left\{{ }^{n} \Phi^{\prime}, n=1,2, \ldots\right\}$ fulfilling (8). Form the sequence ${ }^{1} \Phi,{ }^{1} \Phi^{\prime}$, ${ }^{2} \Phi,{ }^{2} \Phi^{\prime}, \ldots$, which also fulfils (8). According to the above said, the sequence

$$
\int_{0}^{t} 1 \Phi \mathrm{d} W, \quad \int_{0}^{t} 1 \Phi^{\prime} \mathrm{d} W, \int_{0}^{t}{ }^{2} \Phi \mathrm{d} W, \int_{0}^{t}{ }^{2} \Phi^{\prime} \mathrm{d} W, \ldots
$$

has one limit point.

We assumed the interval $[0, T], 0<T<\infty$, to be fixed but arbitrary. If $\Phi=$ $=\left\{\Phi_{t}, t \geqq 0\right\}$ is nonanticipative, and

$$
\int_{0}^{t} \Phi_{s}^{2} \mathrm{~d} s<\infty, \quad t \geqq 0, \text { a.s., }
$$

then $\int_{0}^{t} \Phi \mathrm{d} W$ is defined for $t \geqq 0$, and is continuous with probability 1 . Moreover, if

$$
\int_{0}^{\infty} \Phi_{s}^{2} \mathrm{~d} s<\infty \text { a.s. }
$$

we can without considerable changes use the above construction of the integral also for $T=\infty$. Hence, by the continuity of the integral,

$$
\lim _{t \rightarrow \infty} \int_{0}^{t} \Phi \mathrm{d} W=\int_{0}^{\infty} \Phi \mathrm{d} W \text { a.s. }
$$

if (13) holds.

\subsection{Properties}

Let $\Phi=\left\{\Phi_{t}, t \in[0, T]\right\}$ denote a nonanticipative function satisfying $\int_{0}^{T} \Phi^{2} \mathrm{~d} s<$ $<\infty$ a.s. Next we record main properties of stochastic integrals.

Property 1. $\left\{\int_{0}^{t} \Phi \mathrm{d} W, t \in[0, T]\right\}$ is a continuous nonanticipative function.

Proof. The continuity was established. For $t \in[0, T], \int_{0}^{t} \Phi \mathrm{d} W$ is the limit in probability of a sequence of $\mathscr{F}_{t}$-measurable functions. Moreover, $\mathscr{F}_{t}$ contains all sets of $P$-measure 0 . Thus, $\int_{0}^{t} \Phi \mathrm{d} W$ is $\mathscr{F}_{t}$-measurable, $t \in[0, T]$. This together with continuity implies nonanticipativity according to Theorem 2.1.

\section{Property 2.}

$$
\int_{0}^{t}\left(a \Phi+a^{\prime} \Phi^{\prime}\right) \mathrm{d} W=a \int_{0}^{t} \Phi \mathrm{d} W+a^{\prime} \int_{0}^{t} \Phi^{\prime} \mathrm{d} W, \quad t \in[0, T],
$$

for arbitrary constants $a, a^{\prime}$. 
Property 3. Let

$$
E \int_{0}^{T} \Phi^{2} \mathrm{~d} s<\infty
$$

Then

$$
E\left(\int_{0}^{t} \Phi \mathrm{d} W\right)^{2}=E \int_{0}^{t} \Phi^{2} \mathrm{~d} s, \quad t \in[0, T]
$$

$\left(T=\infty\right.$ is also admitted.) If (14) holds, then $\left\{\int_{0}^{t} \Phi \mathrm{d} W, t \in[0, T]\right\}$ is a martingale. In particular,

$$
E \int_{0}^{t} \Phi \mathrm{d} W=0, \quad t \in[0, T]
$$

Proof. A result analogous to Lemma 5 states that to each $\Phi$ satisfying (14) a sequence of simple functions $\left\{{ }^{n} \Phi, n=1,2, \ldots\right\}$ can be found so that

$$
\lim _{n \rightarrow \infty} E \int_{0}^{T}\left({ }^{n} \Phi-\Phi\right)^{2} \mathrm{~d} s=0
$$

(16) implies (8), and hence,

$$
\mathrm{p} \lim _{n \rightarrow \infty} \int_{0}^{t}{ }^{n} \Phi \mathrm{d} W=\int_{0}^{t} \Phi \mathrm{d} W, \quad t \in[0, T]
$$

Moreover, in virtue of (16),

$$
\lim _{\substack{m \rightarrow \infty \\ n \rightarrow \infty}} E \int_{0}^{T}\left({ }^{m} \Phi-{ }^{n} \Phi\right)^{2} \mathrm{~d} s=0 .
$$

By Lemma 3, (15) holds for simple functions. Consequently,

$$
\lim _{\substack{m \rightarrow \infty \\ n \rightarrow \infty}} E\left(\int_{0}^{t}{ }^{t} \Phi \mathrm{d} W-\int_{0}^{t}{ }^{n} \Phi \mathrm{d} W\right)^{2}=0, \quad t \in[0, T] .
$$

From here and from (17), it follows

$$
\underset{n \rightarrow \infty}{\lim .} \int_{0}^{t}{ }^{n} \Phi \mathrm{d} W=\int_{0}^{t} \Phi \mathrm{d} W, \quad t \in[0, T]
$$

(15) is obtained from

$$
E\left(\int_{0}^{t}{ }^{t} \Phi \mathrm{d} W\right)^{2}=E \int_{0}^{t}{ }^{n} \Phi^{2} \mathrm{~d} s, \quad t \in[0, T], \quad n=1,2, \ldots,
$$

letting $n \rightarrow \infty$, and using (16), (18). 
It is not difficult to verify

$$
E\left\{\int_{0}^{t}{ }^{n} \Phi \mathrm{d} W \mid \mathscr{F}_{s}\right\}=\int_{0}^{s}{ }^{n} \Phi \mathrm{d} W, \quad 0 \leqq s \leqq t \leqq T, \quad n=1,2, \ldots
$$

From (18) and from the continuity of the projection operator $E\left\{\mid \mathscr{F}_{s}\right\}$ (see Property 1.5), it follows

$$
E\left\{\int_{0}^{t} \Phi \mathrm{d} W \mid \mathscr{F}_{s}\right\}=\int_{0}^{s} \Phi \mathrm{d} W, \quad 0 \leqq s \leqq t \leqq T .
$$

Hence, $\left\{\int_{0}^{t} \Phi \mathrm{d} W, t \in[0, T]\right\}$ is a martingale, and $E \int_{0}^{t} \Phi \mathrm{d} W=0, t \in[0, T]$.

(15) can be generalized as follows.

Corollary 1. Let

$$
E \int_{0}^{T} \Phi^{2} \mathrm{~d} t<\infty, \quad E \int_{0}^{T} \Phi^{\prime 2} \mathrm{~d} t<\infty .
$$

Then

$$
E \int_{0}^{t} \Phi \mathrm{d} W \int_{0}^{t} \Phi^{\prime} \mathrm{d} W=E \int_{0}^{t} \Phi \Phi^{\prime} \mathrm{d} s, \quad t \in[0, T]
$$

Proof.

$$
\begin{gathered}
E \int_{0}^{t} \Phi \mathrm{d} W \int_{0}^{t} \Phi^{\prime} \mathrm{d} W=E \frac{1}{4}\left[\left(\int_{0}^{t}\left(\Phi+\Phi^{\prime}\right) \mathrm{d} W\right)^{2}-\right. \\
\left.-\left(\int_{0}^{t}\left(\Phi-\Phi^{\prime}\right) \mathrm{d} W\right)^{2}\right]=E \frac{1}{4}\left[\int_{0}^{t}\left(\Phi+\Phi^{\prime}\right)^{2} \mathrm{~d} s-\int_{0}^{t}\left(\Phi-\Phi^{\prime}\right)^{2} \mathrm{~d} s\right]= \\
=E \int_{0}^{t} \Phi \Phi^{\prime} \mathrm{d} s, \quad t \in[0, T]
\end{gathered}
$$

Property 4. For arbitrary positive numbers $a, b$,

(19) $\quad P\left(\sup _{t \in[0, T]}\left|\int_{0}^{t} \Phi \mathrm{d} W\right| \geqq a\right) \leqq P\left(\int_{0}^{T} \Phi^{2} \mathrm{~d} s \geqq b\right)+2 \exp \left\{-\frac{a^{2}}{2 b}\right\}$.

Proof. (19) was proved for simple functions (Lemma 2), and is obtained in the general case by means of the passage to the limit used to define the integral.

Property 5. Let $\left\{{ }^{n} \Phi, n=1,2, \ldots\right\}$ be a sequence of nonanticipative functions such that

$$
\int_{0}^{T}{ }^{n} \Phi^{2} \mathrm{~d} s<\infty \text { a.s. , } \quad n=1,2, \ldots, \mathrm{p} \lim _{n \rightarrow \infty} \int_{0}^{T}\left({ }^{n} \Phi-\Phi\right)^{2} \mathrm{~d} s=0 .
$$


Then

$$
\mathrm{p} \lim _{n \rightarrow \infty} \sup _{t \in[0, T]}\left|\int_{0}^{t}{ }^{n} \Phi \mathrm{d} W-\int_{0}^{t} \Phi \mathrm{d} W\right|=0 .
$$

Proof. Let $\varepsilon>0, b>0$ be arbitrary. By Property 4

$$
\begin{gathered}
\varlimsup_{n \rightarrow \infty} P\left(\sup _{t \in[0, T]}\left|\int_{0}^{t}{ }^{n} \Phi \mathrm{d} W-\int_{0}^{t} \Phi \mathrm{d} W\right| \geqq \varepsilon\right) \leqq \varlimsup_{n \rightarrow \infty} P\left(\int_{0}^{T}\left({ }^{n} \Phi-\Phi\right)^{2} \mathrm{~d} s \geqq\right. \\
\geqq b)+2 \exp \left\{-\frac{\varepsilon^{2}}{2 b}\right\}=2 \exp \left\{-\frac{\varepsilon^{2}}{2 b}\right\} .
\end{gathered}
$$

The last expression tends to 0 as $b \rightarrow 0$. This proves (20).

Property 6. Let, with probability 1 , the trajectory of $\Phi$ have a continuous derivative $\Phi^{\prime}=\left\{\Phi_{t}^{\prime}, t \in[0, T]\right\},(T<\infty)$. Then

$$
\int_{0}^{T} \Phi \mathrm{d} W=\Phi_{0} W_{T}+\int_{0}^{T} \Phi_{t}^{\prime}\left(W_{T}-W_{t}\right) \mathrm{d} t
$$

Proof.

$$
\begin{gathered}
\int_{0}^{T} \Phi \mathrm{d} W=\mathrm{p} \lim _{n \rightarrow \infty} \sum_{k=0}^{n-1} \Phi_{k T / n}\left(W_{(k+1) T / n}-W_{k T / n}\right)= \\
\mathrm{p} \lim _{n \rightarrow \infty}\left[\Phi_{0} W_{T}+\sum_{k=1}^{n}\left(\Phi_{k T / n}-\Phi_{(k-1) T / n}\right)\left(W_{T}-W_{k T / n}\right)\right]= \\
=\Phi_{0} W_{T}+\int_{0}^{T} \Phi_{t}^{\prime}\left(W_{T}-W_{t}\right) \mathrm{d} t
\end{gathered}
$$

Property 7. Let $\Phi=\left\{\Phi_{t}, t \geqq 0\right\}$ be a nonanticipative function satisfying (12). Let $\tau$ be a stopping time, and let

$$
\int_{0}^{\infty} \chi_{\{\tau>s\}} \Phi_{s}^{2} \mathrm{~d} s<\infty \text { a.s. }
$$

Then

(21)

$$
\int_{0}^{\infty} \chi_{\{r>s\}} \Phi_{s} \mathrm{~d} W_{s}=\int_{0}^{\tau} \Phi_{s} \mathrm{~d} W_{s}
$$

(For $\tau=\infty$ the right-hand side is $\lim _{t \rightarrow \infty} \int_{0}^{t} \Phi \mathrm{d} W$.)

Proof. Note that $\left\{\chi_{\{t>t}, t \geqq 0\right\}$ is nonanticipative. It is right-continuous and complies with $\mathscr{F}$, because $\{\tau>t\} \in \mathscr{F}_{t}, t \geqq 0$, by the definition of the stopping 
time. Take $T>0$. Set $\tau^{\prime}=\tau \wedge T=\min (\tau, T)$. We shall demonstrate

$$
\int_{0}^{\infty} \chi_{\left\{r^{\prime}>s\right\}} \Phi_{s} \mathrm{~d} W_{s}=\int_{0}^{\tau^{\prime}} \Phi_{s} \mathrm{~d} W_{s}
$$

Obviously,

$$
\lim _{T \rightarrow \infty} \int_{0}^{\infty}\left(\chi_{\left\{r^{\prime}>s\right\}} \Phi_{s}-\chi_{\{\tau>s\}} \Phi_{s}\right)^{2} \mathrm{~d} s=0 \text { a.s. }
$$

Hence, (21) follows from (22) as $T \rightarrow \infty$ in virtue of Property 5 and of the continuity of the integral.

Define a sequence of stopping times $\left\{\tau_{n}^{\prime}, n=1,2, \ldots\right\}$ as follows:

$$
\begin{gathered}
\tau_{n}^{\prime}=0 \quad \text { if } \tau^{\prime}=0, \quad \tau_{n}^{\prime}=\frac{k+1}{n} T \text { if } \frac{k}{n} T<\tau^{\prime} \leqq \frac{k+1}{n} T, \\
k=0, \ldots, n-1 .
\end{gathered}
$$

$\left\{\chi_{\left(\tau_{n^{\prime}}>t\right\}}, t \geqq 0\right\}, n=1,2, \ldots$, are simple functions. Further, let $\left\{{ }^{n} \Phi, n=1,2, \ldots\right\}$ be a sequence of simple functions such that

$$
\int_{0}^{T}{ }^{n} \Phi^{2} \mathrm{~d} s<\infty \quad \text { a.s. }, \quad n=1,2, \ldots, \mathrm{p} \lim _{n \rightarrow \infty} \int_{0}^{T}\left({ }^{n} \Phi-\Phi\right)^{2} \mathrm{~d} s=0 .
$$

From (20) it follows

$$
\mathrm{p} \lim _{n \rightarrow \infty} \int_{0}^{\tau_{n^{\prime}}}{ }^{n} \Phi \mathrm{d} W=\int_{0}^{\tau^{\prime}} \Phi \mathrm{d} W .
$$

From the definition of the integral of a simple function it is easily checked that

$$
\int_{0}^{\tau_{n}{ }^{n}} \Phi \mathrm{d} W=\int_{0}^{\infty} \chi_{\left\{\tau_{n^{\prime}}>s\right\}}{ }^{n} \Phi_{s} \mathrm{~d} W_{s}, \quad n=1,2, \ldots
$$

Finally,

$$
\begin{gathered}
\int_{0}^{\infty}\left(\chi_{\left\{t^{\prime}>s\right\}}{ }^{n} \Phi_{s}-\chi_{\left\{\tau^{\prime}>s\right\}} \Phi_{s}\right)^{2} \mathrm{~d} s \leqq \\
\leqq 2\left[\int_{0}^{T}\left({ }^{n} \Phi-\Phi\right)^{2} \mathrm{~d} s+\int_{0}^{T}\left(\chi_{\left\{\tau^{\prime}>s\right\}}-\chi_{\left\{\tau^{\prime}>s\right\}}\right) \Phi_{s}^{2} \mathrm{~d} s\right] \rightarrow 0 \quad \text { in prob. }
\end{gathered}
$$

Hence, according to Property 4,

$$
\mathrm{p} \lim _{n \rightarrow \infty} \int_{0}^{\infty} \chi_{\left\{\tau_{n^{\prime}}>s\right\}}{ }^{n} \Phi_{s} \mathrm{~d} W_{s}=\int_{0}^{\infty} \chi_{\left\{\tau^{\prime}>s\right\}} \Phi_{s} \mathrm{~d} W_{s}
$$

(23), (24), (25) give (22). 


\section{THE ITÔ FORMULA}

\subsection{Proof}

As already mentioned in $\S 1.3$, the calculations with the stochastic differentials follow other rules than the calculations with the ordinary ones. The basic rule, namely the Itô formula, will be derived in the present chapter. Again we suppose given a probability space $(\Omega, \mathscr{A}, P)$ supplied with an increasing family of $\sigma$-algebras $\mathscr{F}=\left\{\mathscr{F}_{t}, t \geqq 0\right\}$ such that $\mathscr{F}_{0}$ contains all $P$-null sets. First we shall treat the onedimensional case, and assume a Wiener process $W=\left\{W_{t}, t \geqq 0\right\}, W_{0} \equiv 0$, defined on $(\Omega, \mathscr{A}, P)$.

Stochastic differentials are defined with aid of integrals.

Definition 1. Random process $X=\left\{X_{t}, t \in[0, T]\right\}$ is an Itô process, if

$$
X_{t}=X_{0}+\int_{0}^{t} A_{s} \mathrm{~d} s+\int_{0}^{t} B_{s} \mathrm{~d} W_{s}, \quad t \in[0, T],
$$

where 1. $X_{0}$ is $\mathscr{F}_{0}$-measurable.

2. $A=\left\{A_{t}, t \in[0, T]\right\}, B=\left\{B_{t}, t \in[0, T]\right\}$ are nonanticipative processes, satisfying

$$
\int_{0}^{T}\left|A_{t}\right| \mathrm{d} t<\infty \quad \text { a.s., } \quad \int_{0}^{T} B_{t}^{2} \mathrm{~d} t<\infty \text { a.s. }
$$

The contents of Definition 1 will be briefly stated by saying that $X$ has stochastic differential

$$
\mathrm{d} X_{t}=A_{t} \mathrm{~d} t+B_{t} \mathrm{~d} W_{t}, \quad t \in[0, T] .
$$

From Definition 1 follows that an Ito process is nonanticipative. A smooth function of Itô processes is itself an Itô process.

Theorem 1. Let random processes ${ }^{i} X=\left\{{ }^{i} X_{t}, t \in[0, T]\right\}, i=1, \ldots, m$, have stochastic differentials

$$
\mathrm{d}^{i} X_{t}={ }^{i} A_{t} \mathrm{~d} t+{ }^{i} B_{t} \mathrm{~d} W_{t}, \quad t \in[0, T], \quad i=1, \ldots, m .
$$

Let $f\left(t, x^{1}, \ldots, x^{m}\right)$ be a function on $[0, T] \times R^{m}$ with continuous derivatives

$$
\begin{gathered}
f=\frac{\partial}{\partial t} f, \quad f_{i}=\frac{\partial}{\partial x^{i}} f, \quad i=1, \ldots, m, \\
f_{i j}=\frac{\partial^{2}}{\partial x^{i} \partial x^{j}} f, \quad i, j=1, \ldots, m,
\end{gathered}
$$


and let $Y_{t}=f\left(t,{ }^{1} X_{t}, \ldots,{ }^{m} X_{t}\right), t \in[0, T]$. Then $Y$ has stochastic differential

$$
\mathrm{d} Y_{t}=f \mathrm{~d} t+\sum_{i=1}^{m} f_{i} \mathrm{~d}{ }^{i} X_{t}+\frac{1}{2} \sum_{i, j=1}^{m} f_{i j} \mathrm{~d}^{i} X_{t} \mathrm{~d}{ }^{j} X_{t}, \quad t \in[0, T],
$$

where $f=f\left(t,{ }^{1} X_{t}, \ldots,{ }^{m} X_{t}\right), \ldots$, and

$$
\mathrm{d}^{i} X_{t} \mathrm{~d}^{j} X_{t}={ }^{i} B_{t}{ }^{j} B_{t} \mathrm{~d} t, \quad t \in[0, T], \quad i, j=1, \ldots, m .
$$

(2) is the Itô formula. It indicates that we have to use Taylor's development up to the second order terms when computing stochastic differentials. To explain (3), let us recall Lemma 1.1, and write

$$
\begin{gathered}
\mathrm{d}^{i} X_{t} \mathrm{~d}^{j} X_{t}={ }^{i} A_{t}{ }^{j} A_{t}(\mathrm{~d} t)^{2}+\left({ }^{i} A_{t}{ }^{j} B_{t}+{ }^{i} B_{t}{ }^{j} A_{t}\right) \mathrm{d} t \mathrm{~d} W_{t}+ \\
+{ }^{i} B_{t}{ }^{j} B_{t}\left(\mathrm{~d} W_{t}\right)^{2}={ }^{i} B_{t}{ }^{j} B_{t} \mathrm{~d} t+\mathrm{o}(\mathrm{d} t) .
\end{gathered}
$$

(2) can be written in the form

$$
\begin{aligned}
\mathrm{d} Y_{t}= & \left(f+\sum_{i}{ }^{i} A_{t} f_{i}+\frac{1}{2} \sum_{i, j}{ }^{i} B_{t}{ }^{j} B_{t} f_{i j}\right) \mathrm{d} t+ \\
& +\left(\sum_{i}{ }^{i} B_{t} f_{i}\right) \mathrm{d} W_{t}, \quad t \in[0, T],
\end{aligned}
$$

consistent with (1). Note that from 2. of Definition 1 and from the continuity of $f, f_{i}, f_{i j}$, it follows

$$
\begin{gathered}
\int_{0}^{T}\left|j+\sum_{i}{ }^{i} A_{t} f_{i}+\frac{1}{2} \sum_{i, j}{ }^{i} B_{t}{ }^{j} B_{t} f_{i j}\right| \mathrm{d} t<\infty, \\
\int_{0}^{T}\left(\sum_{i}{ }^{i} B_{t} f_{i}\right)^{2} \mathrm{~d} t<\infty \quad \text { a.s. }
\end{gathered}
$$

The proof of Theorem 1 will be preceded by a lemma.

Lemma 1. Let $\Phi=\left\{\Phi_{t}, t \in[0, S]\right\},(S<\infty)$, be a nonanticipative random function with continuous trajectories. Then

$$
\mathrm{p} \lim _{n \rightarrow \infty} \sum_{k=0}^{2^{n}-1} \Phi_{k 2-n S}\left(W_{(k+1) 2-n S}-W_{k 2-n S}\right)^{2}=\int_{0}^{S} \Phi_{t} \mathrm{~d} t .
$$

Proof. If $\Phi$ is a simple function,

$$
\Phi_{t}=\varphi_{j}, \quad t_{j} \leqq t<t_{j+1}, \text { where } t_{0}=0<t_{1}<\ldots<t_{h}=S,
$$

then it is not difficult to deduce from Lemma 1.1 that

$$
\lim _{n \rightarrow \infty} \sum_{k=0}^{2 n-1} \Phi_{k 2-n S}\left(W_{(k+1) 2-n S}-W_{k 2-n S}\right)^{2}=\sum_{j=0}^{h-1} \varphi_{j}\left(t_{j+1}-t_{j}\right)=\int_{0}^{S} \Phi_{t} \mathrm{~d} t \quad \text { a.s. }
$$


To prove (4) for arbitrary $\Phi$, one considers simple functions $\left\{\Phi_{[t l] / t}, t \in[0, S]\right\}$, $l=1,2, \ldots$, which approximate $\Phi$ so that

$$
\lim _{l \rightarrow \infty} P\left(\sup _{t \in[0, S]}\left|\Phi_{[t l] / l}-\Phi_{t}\right| \geqq \varepsilon\right)=0, \quad \varepsilon>0 .
$$

Proof of Theorem 1. Let $S \in[0, T]$ be arbitrary. We have to demonstrate

$$
Y_{S}-Y_{0}=\int_{0}^{S}\left(\dot{f}+\sum_{i}{ }^{i} A f_{i}+\frac{1}{2} \sum_{i, j}{ }^{i} B{ }^{j} B f_{i j}\right) \mathrm{d} t+\int_{0}^{S}\left(\sum_{i}{ }^{i} B f_{i}\right) \mathrm{d} W .
$$

The demonstration can be reduced to the case when ${ }^{i} A,{ }^{i} B$ are simple functions. In the general case one takes sequences of simple functions

$$
\left\{{ }_{n}^{i} A, n=1,2, \ldots\right\}, \quad\left\{{ }_{n}^{i} B, n=1,2, \ldots\right\}, i=1, \ldots, m,
$$

such that

$$
\mathrm{p} \lim _{n \rightarrow \infty} \int_{0}^{s}\left|{ }_{n}^{i} A-{ }^{i} A\right| \mathrm{d} t=0, \quad \mathrm{p} \lim \int_{n \rightarrow \infty}^{S}\left({ }_{0}^{i} B-{ }^{i} B\right)^{2} \mathrm{~d} t=0, \quad i=1, \ldots, m .
$$

If (5) holds with ${ }^{i} A,{ }^{i} B$ replaced by ${ }_{n}^{i} A,{ }_{n}^{i} B$, then it remains valid in the limit as $n \rightarrow \infty$. Thus, let ${ }^{i} A,{ }^{i} B, i=1, \ldots, m$, be simple functions. Let us divide $[0, S]$ into subintervals $\left[s, s^{\prime}\right]$, on which these functions are constant,

$$
{ }^{i} A_{t}={ }^{i} \alpha, \quad{ }^{i} B_{t}={ }^{i} \beta, \quad t \in\left[s, s^{\prime}\right], \quad i=1, \ldots, m .
$$

${ }^{i} \alpha,{ }^{i} \beta, i=1, \ldots, m$, are $\mathscr{F}_{s}$-measurable random variables. We have to show that

$$
Y_{s^{\prime}}-Y_{s}=\int_{s}^{s^{\prime}}\left(\dot{f}+\sum_{i}{ }^{i} \alpha f_{i}+\frac{1}{2} \sum_{i, j}^{i} \beta^{j} \beta f_{i j}\right) \mathrm{d} t+\int_{s}^{s^{\prime}}\left(\sum_{i}^{i} \beta f_{i}\right) \mathrm{d} W,
$$

where the arguments of the derivatives are $t$ and

$$
{ }^{i} X_{t}={ }^{i} X_{s}+{ }^{i} \alpha(t-s)+{ }^{i} \beta\left(W_{t}-W_{s}\right), \quad i=1, \ldots, m .
$$

Let

$$
s^{\prime}-s=h, \quad \Delta Y_{s+k 2-n_{h}}=Y_{s+(k+1) 2-n_{h}}-Y_{s+k 2-n_{h}} .
$$

Introducing obvious further denotations, we infer as follows.

$$
\begin{gathered}
Y_{s^{\prime}}-Y_{s}=\lim _{n \rightarrow \infty} \sum_{k=0}^{2^{n-1}} \Delta Y_{s+k 2^{-n_{h}}}=\lim _{n \rightarrow \infty}\left\{\sum _ { k } \left[\dot{f}\left(s+k 2^{-n} h,{ }^{1} X_{s+k 2^{-n_{h}}}, \ldots\right) 2^{-n} h+\right.\right. \\
\left.+\sum_{i} f_{i} \Delta^{i} X_{s+k 2^{-n_{h}}}+\frac{1}{2} \sum_{i, j} f_{i j} \Delta^{i} X_{s+k 2-n_{h}} \Delta^{j} X_{s+k 2-n_{h}}\right]+o(1)+ \\
\left.+o\left(\sum_{i} \sum_{k}\left(\Delta^{i} X_{s+k 2^{-n_{h}}}\right)^{2}\right)\right\}=\mathrm{p} \lim _{n \rightarrow \infty}\left\{\sum_{k}\left[j+\sum_{i} f_{i}{ }^{i} \alpha\right] 2^{-n} h+\right.
\end{gathered}
$$




$$
\begin{aligned}
& \left.+\sum_{k} \sum_{i} f_{i}{ }^{i} \beta \Delta W_{s+k 2-n_{h}}+\frac{1}{2} \sum_{k}\left(\sum_{i, j} f_{i j}{ }^{i} \beta^{j} \beta\right)\left(\Delta W_{s+k 2-n_{h}}\right)^{2}\right\}= \\
& =\int_{s}^{s^{\prime}}\left(f^{i}+\sum_{i}{ }^{i} \alpha f_{i}+\frac{1}{2} \sum_{i, j}{ }^{i} \beta^{j} \beta f_{i j}\right) \mathrm{d} t+\int_{s}^{s^{\prime}}\left(\sum_{i}{ }^{i} \beta f_{i}\right) \mathrm{d} W,
\end{aligned}
$$

in virtue of Lemma 1.

The integral with respect to an Itô process is defined in a straight-forward manner.

Definition 2. Let $X=\left\{X_{t}, t \in[0, T]\right\}$ have stochastic differential (1), and let $\Phi=$ $=\left\{\Phi_{t}, t \in[0, T]\right\}$ be a nonanticipative random function such that

$$
\int_{0}^{T}\left|\Phi_{t} A_{t}\right| \mathrm{d} t<\infty, \quad \int_{0}^{T}\left(\Phi_{t} B_{t}\right)^{2} \mathrm{~d} t<\infty \text { a.s. }
$$

Then

$$
\int_{0}^{t} \Phi_{s} \mathrm{~d} X_{s}=\int_{0}^{t} \Phi_{s} A_{s} \mathrm{~d} s+\int_{0}^{t} \Phi_{s} B_{s} \mathrm{~d} W_{s}, \quad t \in[0, T]
$$

Next we generalize the Itô formula to the case when a multidimensional Wiener process $\bar{W}=\left\{W_{t}=\left({ }^{1} W_{t}, \ldots,{ }^{r} W_{t}\right)^{\prime}, t \geqq 0\right\}$ occurs in the differentials.

Theorem 2. Let random processes ${ }^{i} X=\left\{{ }^{i} X_{t}, t \in[0, T]\right\}, i=1, \ldots, m$, have stochastic differentials

$$
\mathrm{d}^{i} X_{t}={ }^{i} A_{t} \mathrm{~d} t+\sum_{l=1}^{r}{ }^{i l} B_{t} \mathrm{~d}{ }^{l} W_{t}, \quad t \in[0, T], \quad i=1, \ldots, m .
$$

Let $f$ and $Y$ be as in Theorem 1 . Then (2) holds with

$$
\mathrm{d}{ }^{i} X_{t} \mathrm{~d}{ }^{j} X_{t}=\sum_{i=1}^{r}{ }^{i t} B_{t}{ }^{j l} B_{t} \mathrm{~d} t, \quad t \in[0, T], \quad i, j=1, \ldots, m .
$$

As in Definition 1, (6) means that ${ }^{i} X, i=1, \ldots, m$, are nonanticipative, and

$$
{ }^{i} X_{t}={ }^{i} X_{0}+\int_{0}^{t}{ }^{i} A_{s} \mathrm{~d} s+\sum_{i} \int_{0}^{t}{ }^{i t} B_{s} \mathrm{~d}^{l} W_{s}, \quad t \in[0, T], \quad i=1, \ldots, m .
$$

(7) results from the following multiplication rules for differentials

$$
\begin{gathered}
(\mathrm{d} t)^{2}=0, \quad \mathrm{~d} t \mathrm{~d}^{l} W=0, \quad\left(\mathrm{~d}^{l} W\right)^{2}=\mathrm{d} t, \\
\mathrm{~d}^{l} W \mathrm{~d}^{l^{\prime}} W=0, \quad l, l^{\prime}=1, \ldots, r, \quad l \neq l^{\prime} .
\end{gathered}
$$

The proof of Theorem 2 follows the same pattern as the proof of Theorem 1. Only the additional occurence of cross products $\Delta^{l} W_{s+k 2-n_{h}} \Delta^{l} W_{s+k 2-m_{h}}$ requires consideration. This is done in the subsequent lemma. 
Lemma 2. Let $\Phi$ be as in Lemma 1. Set $\Delta^{l} W_{k 2-n S}={ }^{l} W_{(k+1) 2-n S}-{ }^{l} W_{k 2-n S}$. Then

(9) $\quad \lim _{n \rightarrow \infty} \sum_{k=0}^{2^{n}-1} \Phi_{k 2-n_{S}} \Delta^{l} W_{k 2-n_{S}} \Delta^{l^{\prime}} W_{k 2-n_{S}}=0, \quad l, l^{\prime}=1, \ldots, r, \quad l \neq l^{\prime}$.

Proof. To establish (9), one shows first that

$$
\lim _{n \rightarrow \infty} \sum_{k=0}^{2 n-1} \Delta^{l} W_{k 2-n S} \Delta^{l^{\prime}} W_{k 2-n S}=0 \quad \text { a.s., } \quad l \neq l^{\prime} .
$$

(10) is proved with aid of the Borel-Cantelli lemma. Namely, for $\varepsilon>0$,

$$
\begin{gathered}
\sum_{n=1}^{\infty} P\left(\left|\sum_{k=0}^{2^{n}-1} \Delta^{l} W_{k 2-n S} \Delta^{l^{\prime}} W_{k 2-n S}\right| \geqq \varepsilon\right) \leqq \varepsilon^{-2} \sum_{n=1}^{\infty} E\left(\sum_{k=0}^{2^{n}-1} \Delta^{l} W_{k 2-n S} \Delta^{l^{\prime}} W_{k 2-n S}\right)^{2}= \\
=\varepsilon^{-2} \sum_{n=1}^{\infty} \sum_{k=0}^{2^{n-1}} E\left(\Delta^{l} W_{k 2-n S}\right)^{2} E\left(\Delta^{l^{\prime}} W_{k 2-n S}\right)^{2}=\varepsilon^{-2} \sum_{n=1}^{\infty} 2^{n}\left(2^{-n} S\right)^{2}<\infty .
\end{gathered}
$$

Hence, with probability 1 , the sum on the right-hand side of (10) is less than $\varepsilon$ for all sufficiently large $n$.

As in the proof of Lemma 1 it is seen that (9) holds, if $\Phi$ is a simple function, and is proved for arbitrary $\Phi$ using approximation by simple functions.

Lemma 2 explains the last equality in (8). Relation (10) means that the mixed variation of two independent Wiener processes vanishes. Moreover, they yield orthogonal stochastic integrals.

Lemma 3. Let $\Phi=\left\{\Phi_{t}, t \in[0, T]\right\}, \Phi^{\prime}=\left\{\Phi_{t}^{\prime}, t \in[0, T]\right\}$ be nonanticipative and such that

$$
E \int_{0}^{T} \Phi_{t}^{2} \mathrm{~d} t<\infty, \quad E \int_{0}^{T} \Phi_{t}^{\prime 2} \mathrm{~d} t<\infty
$$

Then

$$
E \int_{0}^{t} \Phi \mathrm{d}^{l} W \int_{0}^{t} \Phi^{\prime} \mathrm{d}{ }^{l^{\prime}} W=0, \quad t \in[0, T], \quad l \neq l^{\prime} .
$$

Proof. Remind the proof of Property 3.3. Let $l \neq l^{\prime}$. Take sequences of simple functions $\left\{{ }^{n} \Phi, n=1,2, \ldots\right\},\left\{{ }^{n} \Phi^{\prime}, n=1,2, \ldots\right\}$ such that

$$
\begin{gathered}
\text { l.i.m. } \int_{n \rightarrow \infty}^{t} n \Phi \mathrm{d}^{t} W=\int_{0}^{t} \Phi \mathrm{d}^{t} W \\
\text { l.i.m. } \int_{n \rightarrow \infty}^{t} n \Phi^{\prime} \mathrm{d}^{l^{\prime}} W=\int_{0}^{t} \Phi^{\prime} \mathrm{d}^{l^{\prime}} W, \quad t \in[0, T] .
\end{gathered}
$$


Using the independence of ' $W$ and ${ }^{\prime \prime} W$ it is seen from Definition 3.2 that

$$
E \int_{0}^{t}{ }^{n} \Phi \mathrm{d}^{l} W \int_{0}^{t}{ }^{n} \Phi^{\prime} \mathrm{d}^{l^{\prime}} W=0, \quad t \in[0, T], \quad n=1,2, \ldots
$$

From here and from (12) follows (11).

\subsection{Examples}

Example 1. Let $\left\{X_{t}, t \geqq 0\right\}, X_{0} \neq 0,\left\{Y_{t}, t \geqq 0\right\}$, have stochastic differentials

$$
\mathrm{d} X_{t}=-\sin \Phi_{t} \mathrm{~d} W_{t}, \quad \mathrm{~d} Y_{t}=\cos \Phi_{t} \mathrm{~d} W_{t}, \quad \Phi_{t}=\operatorname{arctg}\left(Y_{t} / X_{t}\right), \quad t \geqq 0 .
$$

Let us compute the differential of $R_{t}^{2}=X_{t}^{2}+Y_{t}^{2}, t \geqq 0$. We have $\mathrm{d}\left(R^{2}\right)=$ $=\mathrm{d}\left(X^{2}+Y^{2}\right)=2 X \mathrm{~d} X+2 Y \mathrm{~d} Y+(\mathrm{d} X)^{2}+(\mathrm{d} Y)^{2}=(-2 X \sin \Phi+2 Y \cos \Phi)$. . $\mathrm{d} W+\left(\sin ^{2} \Phi+\cos ^{2} \Phi\right) \mathrm{d} t=\mathrm{d} t$. We conclude that for $t \geqq 0$ the random point with coordinates $\left(X_{t}, Y_{t}\right)$ lies in the plane at the distance $R_{t}=\sqrt{ }\left(R_{0}+t\right)$ from the origin.

Example 2. Let ${ }^{i} X=\left\{{ }^{i} X_{t}, t \in[0, T]\right\}, i=1,2$, have stochastic differentials

$$
\mathrm{d}{ }^{i} X_{t}={ }^{i} A_{t} \mathrm{~d} t+{ }^{i} B_{t} \mathrm{~d} W_{t}, \quad t \in[0, T] .
$$

From Theorem 1, it follows

$$
\begin{gathered}
\mathrm{d}\left({ }^{1} X^{2} X\right)={ }^{1} X \mathrm{~d}^{2} X+{ }^{2} X \mathrm{~d}^{1} X+\mathrm{d}^{1} X \mathrm{~d}^{2} X={ }^{1} X \mathrm{~d}^{2} X+{ }^{2} X \mathrm{~d}{ }^{1} X+ \\
+{ }^{1} B{ }^{2} B \mathrm{~d} t,
\end{gathered}
$$

or, expressed in integrals,

$$
\int_{0}^{t}{ }^{1} X \mathrm{~d}^{2} X=\left.{ }^{1} X{ }^{2} X\right|_{0} ^{t}-\int_{0}^{t}{ }^{2} X \mathrm{~d}^{1} X-\int_{0}^{t}{ }^{1} B^{2} B \mathrm{~d} s, \quad t \in[0, T] .
$$

This is the formula for partial integration of stochastic integrals.

Example 3. Let $f(x)$ be a continuously differentiable function on $(-\infty, \infty)$. Let us compute $\int_{0}^{t} f(W) \mathrm{d} W$. Denote by $F$ the integral of $f$, i.e. $(\mathrm{d} / \mathrm{d} x) F=f$. Then

$$
\mathrm{d} F(W)=f(W) \mathrm{d} W+\frac{1}{2} f^{\prime}(W) \mathrm{d} t .
$$

Hence,

$$
\int_{0}^{t} f\left(W_{s}\right) \mathrm{d} W_{s}=F\left(W_{t}\right)-F\left(W_{0}\right)-\frac{1}{2} \int_{0}^{t} f^{\prime}\left(W_{s}\right) \mathrm{d} s, \quad t \geqq 0
$$

34 
Example 4. Let $\Phi=\left\{\Phi_{r}, t \in[0, T]\right\}$ be a nonanticipative random function such that $\int_{0}^{T} \Phi^{2} \mathrm{~d} t<\infty$ a.s. Then

$$
Z_{t}=\exp \left\{\int_{0}^{t} \Phi \mathrm{d} W-\frac{1}{2} \int_{0}^{t} \Phi^{2} \mathrm{~d} s\right\}, \quad t \in[0, T] .
$$

is the unique solution of the equation

$$
\mathrm{d} Z_{t}=\Phi_{t} Z_{t} \mathrm{~d} W_{t}, \quad t \in[0, T]
$$

with initial condition $Z_{0} \equiv 1$.

(13) follows from the differentiation

$$
\mathrm{d} Z=Z\left(\Phi \mathrm{d} W-\frac{1}{2} \Phi^{2} \mathrm{~d} t\right)+\frac{1}{2} Z \Phi^{2} \mathrm{~d} t=\Phi Z \mathrm{~d} W .
$$

To prove the unicity, let $Z^{*}=\left\{Z_{t}^{*}, t \in[0, T]\right\}, Z_{0}^{*} \equiv 1$, be also a solution of (13). Differentiate the ratio $Z^{*} / Z$.

$$
\begin{gathered}
\mathrm{d}\left(\frac{Z^{*}}{Z}\right)=\frac{1}{Z} \mathrm{~d} Z^{*}-\frac{Z^{*}}{Z^{2}} \mathrm{~d} Z-\frac{1}{Z^{2}} \mathrm{~d} Z^{*} \mathrm{~d} Z+\frac{Z^{*}}{Z^{3}}(\mathrm{~d} Z)^{2}= \\
=\frac{1}{Z} \Phi Z^{*} \mathrm{~d} W-\frac{Z^{*}}{Z^{2}} \Phi Z \mathrm{~d} W-\frac{1}{Z^{2}} \Phi^{2} Z Z^{*} \mathrm{dt}+\frac{Z^{*}}{Z^{3}} \Phi^{2} Z^{2} \mathrm{~d} t=0 .
\end{gathered}
$$

Hence, $Z_{i}^{*} / Z_{t}=1, t \in[0, T]$, a.s.

In particular, the solution of $\mathrm{d} Z=Z \mathrm{~d} W, Z_{0} \equiv 1$, is $\exp \left\{W_{t}-\frac{1}{2} t\right\}$.

An illustrative application of the Ito formula is provided by the following theorem. Its proof involves a straight-forward use of complex valued random variables.

Theorem 3. Let random process $X=\left\{X_{t}, t \in[0, T]\right\}, X_{0} \equiv 0$, have stochastic differential

$$
\mathrm{d} X_{t}=\sum_{i=1}^{r}{ }^{l} B_{t} \mathrm{~d}^{l} W_{t}, \quad t \in[0, T]
$$

and let with probability 1

$$
\sum_{l=1}^{r}{ }^{t} B_{t}^{2}=1 \text { for almost all } t \in[0, T]
$$

Then $X$ is a Wiener process.

Proof. We have to show that for $0 \leqq t<t^{\prime} \leqq T$

$$
E\left\{\exp \left\{\mathrm{i} z\left(X_{t^{\prime}}-X_{t}\right)\right\} \mid \tilde{F}_{t}\right\}=\exp \left\{-\frac{1}{2} z^{2}\left(t^{\prime}-t\right)\right\}, \quad z \in(-\infty, \infty) .
$$

On the right-hand side stands the characteristic function of the normal distribution with mean 0 and variance $t^{\prime}-t$. Thus, (16) states that $X_{t^{\prime}}-X_{t}$ has this distribution, and is independent of $\mathscr{F}_{t}$. 
Denote $Y_{u}=\exp \left\{i z\left(X_{u}-X_{t}\right)\right\}, u \in[t, T]$. Its differential is

$$
\mathrm{d} Y_{u}=\mathrm{i} z Y_{u} \mathrm{~d} X_{u}-\frac{1}{2} z^{2} Y_{u} \sum_{l=1}^{r}{ }^{l} B_{u}^{2} \mathrm{~d} u, \quad u \in[t, T]
$$

Hence, because of (15),

$$
Y_{t^{\prime}}-1=\mathrm{i} z \sum_{i=1}^{r} \int_{t}^{t^{\prime}} Y_{u}{ }^{l} B_{u} \mathrm{~d}^{l} W_{u}-\frac{1}{2} z^{2} \int_{t}^{t^{\prime}} Y_{u} \mathrm{~d} u, t^{\prime} \in[t, T] .
$$

The conditional exception of the first term on the right is 0 by Property 3.3. Consequently,

$$
E\left\{Y_{t^{\prime}} \mid \mathscr{F}_{t}\right\}=1-\frac{1}{2} z^{2} \int_{t}^{t^{\prime}} E\left\{Y_{u} \mid \mathscr{F}_{t}\right\} \mathrm{d} u, \quad t^{\prime} \in[t, T], \text { a.s. }
$$

We see that with probability $1 E\left\{Y_{t^{\prime}} \mid \mathscr{F}_{t}\right\}$ fulfils an integral equation, which has unique solution $\exp \left\{-\frac{1}{2} z^{2}\left(t^{\prime}-t\right)\right\}$.

Corollary 1. Let $X=\left\{X_{t}, t \in[0, T]\right\}$ have stochastic differential (14), and let with probability 1

$$
\sum_{i=1}^{r}{ }^{l} B_{t}^{2}>0 \text { for almost all } t \in[0, T]
$$

Then

$$
\mathrm{d} X_{t}=\left(\sum_{l=1}^{r} B_{t}^{2}\right)^{1 / 2} \mathrm{~d} W_{t}^{*}, \quad t \in[0, T],
$$

where $W^{*}=\left\{W_{t}^{*}, t \in[0, T]\right\}$ is a Wiener process.

Proof. Define

$$
W_{t}^{*}=\int_{0}^{t}\left(\sum_{l=1}^{r}{ }^{l} B_{s}^{2}\right)^{-1 / 2} \mathrm{~d} X_{s}, \quad t \in[0, T] .
$$

Then $W^{*}$ fulfils the hypotheses of Theorem 3, and (17) holds.

\section{STOCHASTIC DIFFERENTIAL EQUATIONS}

\subsection{Linear equations}

Let us begin by considering as an example the model of free nutation proposed in [1]. The rotation axis of the Earth intersects the plane tangent to the Earth at the (North or South) Pole in a point, which performs a random movement containing a periodic component with period one year. This component being eliminated, the 
so called Chandler's movement remains. Let $\bar{X}=\left\{\left({ }^{1} X_{t},{ }^{2} X_{t}\right)^{\prime}, t \geqq t_{0}\right\}$ denote its coordinates as functions of time. $\bar{X}$ has a period of about 14 months with slow fluctuations of amplitude (waves of length $10-20$ years). It was suggested to underlay the empirical studies with a mathematical assumption about the differentials of $\bar{X}$. Namely,

or

$$
\begin{aligned}
& \mathrm{d}^{1} X_{t}=-l^{1} X_{t} \mathrm{~d} t-k^{2} X_{t} \mathrm{~d} t+b \mathrm{~d}^{1} W_{t}, \\
& \mathrm{~d}^{2} X_{t}=k^{1} X_{t} \mathrm{~d} t-l^{2} X_{t} \mathrm{~d} t+b \mathrm{~d}^{2} W_{t}, \quad t \geqq t_{0},
\end{aligned}
$$

$$
\mathrm{d} \bar{X}_{t}=A \bar{X}_{t} \mathrm{~d} t+B \mathrm{~d} \bar{W}_{t}, \quad t \geqq t_{0}
$$

In (1)

$$
A=\left(\begin{array}{rr}
-l, & -k \\
k, & -l
\end{array}\right), \quad B=\left(\begin{array}{ll}
b, & 0 \\
0, & b
\end{array}\right)
$$

$l, k, b$ are positive constants, $\bar{W}=\left\{\bar{W}_{t}=\left({ }^{1} W_{t},{ }^{2} W_{t}\right)^{\prime}, t \geqq t_{0}\right\}$ is a two-dimensional Wiener process.

(1) is a stochastic differential equation for $\bar{X}$, a linear one. Such equations will be dealt with in the present section. In the entire chapter we assume that an $r$-dimensional Wiener process $\bar{W}=\left\{\bar{W}_{t}=\left({ }^{1} W_{t}, \ldots,{ }^{r} W_{t}\right)^{\prime}, t \geqq 0\right\}$ with respect to $\mathscr{F}=\left\{\mathscr{F}_{t}\right.$, $t \geqq 0\}$ is given on a basic space $(\Omega, \mathscr{A}, P)$. $\mathscr{F}_{0}$ contains all sets of $P$-measure zero. We recall that ' indicates the transposition.

First we define the concept of a stochastic differential equation. Let

$$
\begin{gathered}
\bar{a}(t, \bar{x})=\left(a^{1}(t, \bar{x}), \ldots, a^{m}(t, \bar{x})\right)^{\prime}, \quad B(t, \bar{x})=\left\|b_{i j}(t, \bar{x})\right\|_{i=1, j=1}^{m} r, \\
(t, \bar{x}) \in[0, T] \times R^{m},
\end{gathered}
$$

be a Borel measurable vector function, and a Borel measurable matrix function of type $m \times r$, respectively. Let $T<\infty$, for the sake of definiteness. Let the symbol $\bar{X}=\left\{\bar{X}_{t}=\left({ }^{1} X_{t}, \ldots,{ }^{m} X_{t}\right)^{\prime}, t \in[0, T]\right\}$ denote an $m$-dimensional random process. The relation among differentials

$$
\mathrm{d} \bar{X}_{t}=\bar{a}\left(t, \bar{X}_{t}\right) \mathrm{d} t+B\left(t, \bar{X}_{t}\right) \mathrm{d} \bar{W}_{t}, \quad t \in[0, T],
$$

is called a stochastic differential equation.

Definition 1. Let $\bar{\xi}$ be an $\mathscr{F}_{0}$-measurable $m$-dimensional random variable. Random process $\bar{X}=\left\{\bar{X}_{t}, t \in[0, T]\right\}$ is a solution of equation (3) with initial condition $\xi$, if it has stochastic differential (3), and $\bar{X}_{0}=\xi$. If, in addition, any solution of (3), with initial condition $\xi$ is indistinguishable from $\bar{X}$, then $\bar{X}$ is called unique solution.

Specialize now to linear equations. Let

$$
\bar{a}(t, \bar{x})=\bar{a}(t)+A(t) \bar{x}, \quad B(t, \bar{x})=B(t), \quad(t, \bar{x}) \in[0, T] \times R^{m},
$$


where

$$
\begin{gathered}
\bar{a}(t)=\left(a^{1}(t), \ldots, a^{m}(t)\right)^{t}, \quad A(t)=\left\|a_{i j}(t)\right\|_{i, j=1}^{m}, \\
B(t)=\left\|b_{i j}(t)\right\|_{i=1, j=1}^{m},
\end{gathered}
$$

are Borel measurable functions on $[0, T]$. Then (3) becomes

$$
\mathrm{d} \bar{X}_{t}=\left(\vec{a}(t)+A(t) \bar{X}_{t}\right) \mathrm{d} t+B(t) \mathrm{d} \bar{W}_{t}, \quad t \in[0, T] .
$$

Methods of ordinary linear differential equations can be used to investigate the stochastic ones, as it is seen on the subsequent theorem.

Theorem 1. Let $\bar{a}, A, B$ fulfil

$$
\begin{gathered}
\int_{0}^{T}\left|a^{i}(t)\right| \mathrm{d} t<\infty, \int_{0}^{T}\left|a_{i j}(t)\right| \mathrm{d} t<\infty, \int_{0}^{T} b_{i k}(t)^{2} \mathrm{~d} t<\infty, \\
i, j=1, \ldots, m, \quad k=1, \ldots, r .
\end{gathered}
$$

Let $\xi$ be an $\mathscr{F}_{0}$-measurable $m$-dimensional random variable. Then equation (4) with initial condition $\bar{\xi}$ has unique solution

(6) $\quad \bar{X}_{t}=F(t)\left(\bar{\xi}+\int_{0}^{t} F(s)^{-1} \bar{a}(s) \mathrm{d} s+\int_{0}^{t} F(s)^{-1} B(s) \mathrm{d} \bar{W}_{s}\right), \quad t \in[0, T]$,

where $F$ is the fundamental matrix of the equation $(\mathrm{d} / \mathrm{d} t) F=A(t) F$.

Proof. $F$ is the solution of $(\mathrm{d} / \mathrm{d} t) F=A(t) F$ satisfying $F(0)=I, I$ is the unit matrix. From the theory of differential equations it is known that $F$ is uniquely defined and invertible on $[0, T]$. Consequently, $\bar{X}$ is well determined by (6), the integrals exist in virtue of (5). Computing the stochastic differential of $\bar{X}$, one gets from (6)

$$
\begin{gathered}
\mathrm{d} \bar{X}_{t}=A(t) F(t) \mathrm{d} t\left(\xi+\int_{0}^{t} F(s)^{-1} \bar{a}(s) \mathrm{d} s+\int_{0}^{t} F(s)^{-1} B(s) \mathrm{d} \bar{W}_{s}\right)+ \\
+F(t) F(t)^{-1} \bar{a}(t) \mathrm{d} t+F(t) F(t)^{-1} B(t) \mathrm{d} \bar{W}_{t}=\left(\bar{a}(t)+A(t) \bar{X}_{t}\right) \mathrm{d} t+B(t) \mathrm{d} \bar{W}_{t} .
\end{gathered}
$$

Hence, (4) holds. Moreover, $\bar{X}_{0}=\bar{\xi}$, since $F(0)=I$.

To establish the unicity, let $\bar{X}^{*}$ be also a solution of (4) with initial condition $\bar{\xi}$. Then

(7) $\quad \mathrm{d}\left(\bar{X}_{t}-\bar{X}_{t}^{*}\right)=A(t)\left(\bar{X}_{t}-\bar{X}_{t}^{*}\right) \mathrm{d} t, \quad t=[0, T], \quad \bar{X}_{0}-\bar{X}_{0}^{*} \equiv 0$.

A linear differential equation with initial condition 0 has only a trivial solution. Thus, from (7), we conclude that $\bar{X}_{t}=\bar{X}_{t}^{*}, t \in[0, T]$, a.s. 
Differential equations for the mean value and for the covariance (matrix) function are obtainable from (6). Let $E|\bar{\xi}|^{2}<\infty$. Introduce

$$
\begin{gathered}
\bar{e}(t)=E \bar{X}_{t}, R(u, t)=E\left(\bar{X}_{u}-\bar{e}(u)\right)\left(\bar{X}_{t}-\bar{e}(t)\right)^{\prime}, \quad R(t, t)=Q(t), \\
u, t \in[0, T] .
\end{gathered}
$$

From (6) and from Property 3.3, it follows

$$
\ddot{e}(t)=F(t)\left(E \dot{\xi}+\int_{0}^{t} F(s)^{-1} \bar{a}(s) \mathrm{d} s\right), \quad t \in[0, T]
$$

and hence,

$$
\frac{\mathrm{d}}{\mathrm{d} t} \bar{e}=A(t) \bar{e}+\bar{a}(t), \quad t \in[0, T], \quad \bar{e}(0)=E \bar{\xi} .
$$

Further, from (6) one gets for $u \in[t, T]$

$$
\bar{X}_{u}=F(u)\left(F(t)^{-1} \bar{X}_{t}+\int_{t}^{u} F(s)^{-1} \bar{a}(s) \mathrm{d} s+\int_{t}^{u} F(s)^{-1} B(s) \mathrm{d} \overline{W_{s}}\right) .
$$

Thus,

$$
R(u, t)=E \bar{X}_{u}\left(\bar{X}_{t}-\bar{e}(t)\right)^{\prime}=F(u) F(t)^{-1} E \bar{X}_{t}\left(\bar{X}_{t}-\bar{e}(t)\right)^{\prime}=F(u) F(t)^{-1} Q(t),
$$

or

$$
\frac{\mathrm{d}}{\mathrm{d} u} R=A(u) R, \quad u \in[t, T], \quad R(t, t)=Q(t)
$$

Before deriving an equation for $Q$, let us state a lemma.

Lemma 1. Let $G(t)=\left\|g_{i j}(t)\right\|_{i=1, j=1}^{m r}$ be a Borel measurable matrix function on $[0, T]$ such that $\int_{0}^{T} g_{i j}(t)^{2} \mathrm{~d} t<\infty, i=1, \ldots, m, j=1, \ldots, r$. Then

$$
E \int_{0}^{t} G(s) \mathrm{d} \bar{W}_{s}\left(\int_{0}^{t} G(s) \mathrm{d} \bar{W}_{s}\right)^{\prime}=\int_{0}^{t} G(s) G(s)^{\prime} \mathrm{d} s, \quad t \in[0, T] .
$$

Proof. Consider the element on position $i j$ of the matrix on the left. From Lemma 4.3 and from Corollary 3.1, it follows

$$
E\left(\sum_{k} \int_{0}^{t} g_{i k} \mathrm{~d}^{k} W\right)\left(\sum_{l} \int_{0}^{t} g_{j l} \mathrm{~d}^{l} W\right)=\sum_{k} E \int_{0}^{t} g_{i k} \mathrm{~d}^{k} W \int_{0}^{t} g_{j k} \mathrm{~d}^{k} W=\sum_{k} \int_{0}^{t} g_{i k} g_{j k} \mathrm{~d} s .
$$

The last term is the element on position $i j$ of the matrix on the right of $(10)$. 
Using (6) and Lemma 1 we get

$$
\begin{aligned}
Q(t)= & E F(t)\left(\bar{X}_{0}-\tilde{e}(0)+\int_{0}^{t} F(s)^{-1} B(s) \mathrm{d} \bar{W}_{s}\right)\left(\bar{X}_{0}-\bar{e}(0)+\right. \\
& \left.+\int_{0}^{t} F(s)^{-1} B(s) \mathrm{d} \bar{W}_{s}\right)^{\prime} F(t)^{\prime}=F(t) Q(0) F(t)^{\prime}+ \\
& +F(t) \int_{0}^{t} F(s)^{-1} B(s) B(s)^{\prime} F(s)^{-1 \prime} \mathrm{d} s F(t)^{\prime}
\end{aligned}
$$

From here,

$$
\begin{gathered}
\frac{\mathrm{d}}{\mathrm{d} t} Q=A(t) Q+Q A(t)^{\prime}+B(t) B(t)^{\prime}, \quad t \in[0, T] \\
Q(0)=E(\bar{\xi}-E \xi)(\xi-E \bar{\xi})^{\prime} .
\end{gathered}
$$

If $\xi$ has normal distribution, then $\bar{X}$ is a Gaussian process. Its probability distribution is completely defined by $\bar{e}$ and $R$, obtainable from (8), (9), and (11).

Example 1. Let us calculate the covariance function of process $\bar{X}$ satisfying equation (1). Set $t_{0}=0$. The fundamental matrix is

$$
F(t)=\mathrm{e}^{-l t}\left(\begin{array}{rr}
\cos k t, & -\sin k t \\
\sin k t, & \cos k t
\end{array}\right), \quad t \geqq 0 .
$$

Hence,

$$
\begin{aligned}
& { }^{1} X_{t}=\mathrm{e}^{-l t}\left(\xi^{1} \cos k t-\xi^{2} \sin k t\right)+b \int_{0}^{t} \mathrm{e}^{-l(t-s)} \cos k(t-s) \mathrm{d}^{1} W_{s}- \\
& -b \int_{0}^{t} \mathrm{e}^{-l(t-s)} \sin k(t-s) \mathrm{d}^{2} W_{s}, \\
& { }^{2} X_{t}=\mathrm{e}^{-l t}\left(\xi^{1} \sin k t+\xi^{2} \cos k t\right)+b \int_{0}^{t} \mathrm{e}^{-l(t-s)} \sin k(t-s) \mathrm{d}^{1} W_{s}+ \\
& +b \int_{0}^{t} \mathrm{e}^{-l(t-s)} \cos k(t-s) \mathrm{d}^{2} W_{s} .
\end{aligned}
$$

Equation (11) for $Q(t)=\left\|q_{i j}(t)\right\|_{i, j=1}^{2}$ provides the following system of equations for three unknown functions $q_{11}(t), q_{22}(t), q_{12}(t)=q_{21}(t)$ :

$$
\frac{\mathrm{d}}{\mathrm{d} t} q_{11}=-2 l q_{11} \quad-2 k q_{12}+b^{2},
$$

40 


$$
\begin{array}{lll}
\frac{\mathrm{d}}{\mathrm{d} t} q_{22}= & -2 l q_{22} & +2 k q_{12}+b^{2}, \\
\frac{\mathrm{d}}{\mathrm{d} t} q_{12}=k q_{11} & -k q_{22} & -2 l q_{12}, \quad t \geqq 0 .
\end{array}
$$

The soiution is

$q_{11}(t)=\frac{b^{2}}{2 l}\left(1-\mathrm{e}^{-2 l t}\right)+\mathrm{e}^{-2 l t}\left(q_{11}(0) \cos ^{2} k t+q_{22}(0) \sin ^{2} k t-q_{12}(0) \sin 2 k t\right)$,

$q_{22}(t)=\frac{b^{2}}{2 l}\left(1-\mathrm{e}^{-2 l t}\right)+\mathrm{e}^{-2 l t}\left(q_{11}(0) \sin ^{2} k t+q_{22}(0) \cos ^{2} k t+q_{12}(0) \sin 2 k t\right)$,

$q_{12}(t)=q_{21}(t)=\mathrm{e}^{-2 i t}\left(\frac{1}{2} q_{11}(0) \sin 2 k t-\frac{1}{2} q_{22}(0) \sin 2 k t+q_{12}(0) \cos 2 k t\right), t \geqq 0$,

where $Q(0)=E(\bar{\xi}-E \bar{\xi})(\bar{\xi}-E \bar{\xi})^{\prime}$.

Finally, from (9), $R(u, t)=F(u-t) Q(t), u \geqq t \geqq 0$, or

$$
\begin{aligned}
& r_{11}(u, t)=q_{11}(t) \cos k(u-t)-q_{12}(t) \sin k(u-t), \\
& r_{22}(u, t)=q_{12}(t) \sin k(u-t)+q_{22}(t) \cos k(u-t), \\
& r_{12}(u, t)=q_{12}(t) \cos k(u-t)-q_{22}(t) \sin k(u-t), \\
& r_{21}(u, t)=q_{11}(t) \sin k(u-t)+q_{12}(t) \cos k(u-t), \quad u \geqq t \geqq 0 .
\end{aligned}
$$

\subsection{Nonlinear Equations}

Random process $\bar{X}=\left\{\bar{X}_{t}, t \in[0, T]\right\}$ is a diffusion process, if it has stochastic differential

$$
\mathrm{d} \bar{X}_{t}=\bar{a}\left(t, \bar{X}_{t}\right) \mathrm{d} t+B\left(t, \bar{X}_{t}\right) \mathrm{d} \bar{W}_{t}, \quad t \in[0, T],
$$

where $\bar{a}, B$ are as in (2). In applications of diffusion processes it is often possible to obtain functions $\bar{a}, B$ from physical considerations. Thus, the question arises, whether a process with the prescribed differential exists, and in what sense it is unique.

The Euclidean norm of a vector $\bar{m}$ or of a matrix $M$ will be denoted by $|\bar{m}|$ and $|M|$, respectively. Next theorem comes from K. Itô $(1946,1951)$. Successive approximations used in its proof are an analogy of Picard's method from the theory of ordinary differential equations. We keep $T<\infty$.

Theorem 2. Let $\bar{\xi}$ be an $\mathscr{F}_{0}$-measurable $m$-dimensional random variable, $E|\xi|^{2}<\infty$. Let functions $\vec{a}, B$ be as in (2), and fulfil

$$
\begin{gathered}
\text { (13) }|\bar{a}(t, \bar{x})-\bar{a}(t, \bar{y})|+|B(t, \bar{x})-B(t, \bar{y})| \leqq k_{1}|\bar{x}-\bar{y}|, \\
\text { (14) }|\bar{a}(t, \bar{x})|+|B(t, \bar{x})| \leqq k_{2}(1+|\bar{x}|), \quad \bar{x}, \bar{y} \in R^{m}, \quad t \in[0, T],
\end{gathered}
$$


where $k_{1}, k_{2}$ are constants. Then stochastic differential equation (12) has unique solution $\bar{X}=\left\{\bar{X}_{t}, t \in[0, T]\right\}$. Moreover,

$$
E\left|\bar{X}_{t}\right|^{2}<\text { const., } t \in[0, T] .
$$

Proof. 1 . Set ${ }^{0} \bar{X}_{t}=\bar{\xi}, t \in[0, T]$,

(16) ${ }^{n+1} \bar{X}_{t}=\bar{\xi}+\int_{0}^{t} \vec{a}\left(s,{ }^{n} \bar{X}_{s}\right) \mathrm{d} s+\int_{0}^{t} B\left(s,{ }^{n} \bar{X}_{s}\right) \mathrm{d} \bar{W}_{s}, \quad t \in[0, T], n=0,1, \ldots$

The integrals in (16) exist. Further denote

$$
d^{n}(t)=\left.E\right|^{n+1} \bar{X}_{t}-\left.{ }^{n} \bar{X}_{t}\right|^{2}, \quad t \in[0, T], \quad n=0,1, \ldots,
$$

and consider $d^{\circ}(t)$. It holds

$$
d^{0}(t) \leqq E\left(2\left|\int_{0}^{t} \bar{a}(s, \bar{\xi}) \mathrm{d} s\right|^{2}+2\left|\int_{0}^{t} B(s, \bar{\xi}) \mathrm{d} \bar{W}_{s}\right|^{2}\right)
$$

By Lemma 4.3 and Property 3.3,

$$
E\left|\int_{0}^{t} B \mathrm{~d} \bar{W}\right|^{2}=\sum_{i} E\left(\int_{0}^{t} \sum_{j} b_{i j} \mathrm{~d}^{j} W\right)^{2}=\sum_{i} \sum_{j} E \int_{0}^{t} b_{i j}^{2} \mathrm{~d} s=E \int_{0}^{t}|B|^{2} \mathrm{~d} s,
$$

provided that the last term is finite. Hence, using Schwarz inequality and (14), we get

$$
d^{0}(t) \leqq 2 E t \int_{0}^{t}|\bar{a}|^{2} \mathrm{~d} s+2 E \int_{0}^{t}|B|^{2} \mathrm{~d} s \leqq 2 \max (1, T) E 2 k_{2}^{2}\left(1+|\bar{\xi}|^{2}\right) T=l_{0}<\infty .
$$

Similarly, from (16) and (13),

$$
\begin{gathered}
d^{n}(t) \leqq 2 \max (1, T) E\left(\int_{0}^{t}\left|\bar{a}\left(s,{ }^{n} \bar{X}_{s}\right)-\bar{a}\left(s,{ }^{n-1} \bar{X}_{s}\right)\right|^{2} \mathrm{~d} s+\right. \\
\left.+\int_{0}^{t}\left|B\left(s,{ }^{n} \bar{X}_{s}\right)-B\left(s,{ }^{n-1} \bar{X}_{s}\right)\right|^{2} \mathrm{~d} s\right)=\left.2 \max (1, T) k_{1}^{2} \int_{0}^{t} E\right|^{n} \bar{X}_{s}-\left.{ }^{n-1} \bar{X}_{s}\right|^{2} \mathrm{~d} s= \\
=l \int_{0}^{t} d^{n-1}(s) \mathrm{d} s, \quad t \in[0, T], \quad n=1,2, \ldots
\end{gathered}
$$

From here, it follows with aid of induction

$$
d^{n}(t) \leqq l_{0} \frac{(l t)^{n}}{n !}, \quad t \in[0, T], \quad n=0,1, \ldots
$$

42 
2. Let us estimate the differences of the successive terms in (16). Set

$$
\begin{gathered}
\int_{0}^{t}\left(\bar{a}\left(s,{ }^{n} \bar{X}_{s}\right)-\bar{a}\left(s,{ }^{n-1} \bar{X}_{s}\right)\right) \mathrm{d} s=\int_{0}^{t}{ }^{n} \bar{\delta} \mathrm{d} s, \\
\int_{0}^{t}\left(B\left(s,{ }^{n} \bar{X}_{s}\right)-B\left(s,{ }^{n-1} \bar{X}_{s}\right)\right) \mathrm{d} \bar{W}_{s}=\int_{0}^{t}{ }^{n} \Delta \mathrm{d} \bar{W}, \quad t \in[0, T], \quad n=1,2, \ldots
\end{gathered}
$$

We have for $\varepsilon>0$

$$
\begin{aligned}
& P\left(\sup _{t \in[0, T]}\left|\int_{0}^{t}{ }^{n} \bar{\delta} \mathrm{d} s\right| \geqq \varepsilon\right) \leqq P\left(\int_{0}^{T}|n \bar{\delta}| \mathrm{d} s \geqq \varepsilon\right) \leqq \varepsilon^{-2} E\left(\int_{0}^{T}|n \bar{\delta}| \mathrm{d} s\right)^{2} \leqq \\
& \leqq \varepsilon^{-2} T E \int_{0}^{T}|n \bar{\delta}|^{2} \mathrm{~d} s \leqq \varepsilon^{-2} T k_{1}^{2} \int_{0}^{T} d^{n-1}(s) \mathrm{d} s \leqq \frac{T k_{1}^{2} l_{0}}{\varepsilon^{2} l} \frac{(l T)^{n}}{n !}, \quad n=1,2, \ldots
\end{aligned}
$$

The second integral is estimated with aid of the submartingale inequality (Theorem 2.2).

$$
\begin{gathered}
P\left(\sup _{t \in[0, T]}\left|\int_{0}^{t}{ }^{n} \Delta \mathrm{d} \bar{W}\right| \geqq \varepsilon\right) \leqq \varepsilon^{-2} E\left|\int_{0}^{T} n \Delta \mathrm{d} \bar{W}\right|^{2}=\varepsilon^{-2} E \int_{0}^{T}|n \Delta|^{2} \mathrm{~d} s \leqq \\
\leqq \varepsilon^{-2} k_{1}^{2} \int_{0}^{T} d^{n-1}(s) \mathrm{d} s \leqq \frac{k_{1}^{2} l_{0}}{\varepsilon^{2} l} \frac{(l T)^{n}}{n !}, \quad n=1,2, \ldots
\end{gathered}
$$

From (16) we conclude that

$$
P\left(\sup _{t \in[0, T]}\left|{ }^{n+1} \bar{X}_{t}-{ }^{n} \bar{X}_{t}\right| \geqq 2 \varepsilon\right) \leqq \frac{(T+1) k_{1}^{2} l_{0}}{\varepsilon^{2} l} \frac{(l T)^{n}}{n !} \quad n=1,2, \ldots
$$

Setting $\varepsilon_{n}=\left((l T)^{n} /(n-2) !\right)^{1 / 2}, \quad n=2,3, \ldots$, we get

$$
P\left(\sup _{t \in[0, T]}\left|{ }^{n+1} \bar{X}_{t}-{ }^{n} \bar{X}_{t}\right| \geqq 2 \varepsilon_{n}\right) \leqq \frac{\text { const. }}{n(n-1)}, \quad n=2,3, \ldots
$$

From the Borell-Cantelli lemma and from $\sum_{n=2}^{\infty} \varepsilon_{n}<\infty$ it follows that there exists a random process $\bar{X}=\left\{\bar{X}_{t}, t \in[0, T]\right\}$ to which ${ }^{n} \bar{X}, n=0,1, \ldots$, converge with probability 1 uniformly. In symbols,

$$
{ }^{n} \bar{X}_{t} \rightarrow \bar{X}_{t}, \quad t \in[0, T], \text { a.s. }
$$

It follows from (13) that

$$
\bar{a}\left(t,{ }^{n} \bar{X}_{t}\right) \rightarrow \bar{a}\left(t, \bar{X}_{t}\right), \quad B\left(t,{ }^{n} \bar{X}_{t}\right) \rightarrow B\left(t, \bar{X}_{t}\right), \quad t \in[0, T], \quad \text { a.s. }
$$


Letting $n \rightarrow \infty$ in (16) and using Property 3.5, we obtain

$$
\bar{X}_{\mathrm{t}}=\bar{\xi}+\int_{0}^{t} \vec{a}\left(s, \bar{X}_{s}\right) \mathrm{d} s+\int_{0}^{t} B\left(s, \bar{X}_{s}\right) \mathrm{d} \bar{W}_{s}, \quad t \in[0, T] .
$$

Consequently, $\bar{X}$ is a solution of (12) with initial condition $\xi$. Further, we have the estimate

$$
\begin{aligned}
\sqrt{E}\left|\bar{X}_{r}\right|^{2} & =\sqrt{ } E\left|\bar{\xi}+\sum_{n=0}^{\infty}\left({ }^{n+1} \bar{X}_{t}-{ }^{n} \bar{X}_{t}\right)\right|^{2} \leqq \sqrt{ } E|\xi|^{2}+\sum_{n=0}^{\infty} \sqrt{ } d^{n}(t) \leqq \\
& \leqq \sqrt{ } E|\bar{\xi}|^{2}+\sqrt{ } l_{0} \sum_{n=0}^{\infty} \sqrt{ }\left((l T)^{n} / n !\right)<\infty, \quad t \in[0, T] .
\end{aligned}
$$

Hence, (15) is valid.

3. It remains to establish the unicity of $\bar{X}$. Let $\bar{X}^{*}=\left\{\bar{X}_{t}^{*}, t \in[0, T]\right\}$ be also a solution of (12) with initial condition $\vec{\xi}$. Assume in addition

$$
E\left|\bar{X}_{t}^{*}\right|^{2} \leqq \text { const., } \quad t \in[0, T] .
$$

Then

$\bar{X}_{t}^{*}-\bar{X}_{t}=\int_{0}^{t}\left(\bar{a}\left(s, \bar{X}_{s}^{*}\right)-\bar{a}\left(s, \bar{X}_{s}\right)\right) \mathrm{d} s+\int_{0}^{t}\left(B\left(s, \bar{X}_{s}^{*}\right)-B\left(s, \bar{X}_{s}\right)\right) \mathrm{d} \bar{W}_{s}, \quad t \in[0, T]$.

As in Part 1 of the proof we get

$$
E\left|\bar{X}_{t}^{*}-\bar{X}_{t}\right|^{2} \leqq l \int_{0}^{t} E\left|\bar{X}_{s}^{*}-\bar{X}_{s}\right|^{2} \mathrm{~d} s, \quad t \in[0, T] .
$$

Since $E\left|\bar{X}_{t}^{*}-\bar{X}_{t}\right|^{2} \leqq$ const., $t \in[0, T]$, a recursive substitution into (18) gives

$$
E\left|\bar{X}_{t}^{*}-\bar{X}_{t}\right|^{2} \leqq \text { const. }(l t)^{n} / n !, \quad n=0,1, \ldots
$$

We see that $E\left|\bar{X}_{t}^{*}-\bar{X}_{t}\right|^{2}=0, t \in[0, T]$. Moreover $\bar{X}^{*}$ and $\bar{X}$ have with probability 1 continuous trajectories. Therefore

$$
\bar{X}_{t}^{*}=\bar{X}_{t}, \quad t \in[0, T], \quad \text { a.s. }
$$

If (17) does not hold, then we introduce stopping times

$$
\tau_{n}=\inf \left\{t:\left|\bar{X}_{t}^{*}\right| \geqq n\right\}, \quad n=1,2, \ldots
$$

(The infimum of a void set is $\infty$ ) As above we prove $\bar{X}_{t \wedge \tau_{n}}^{*}=\bar{X}_{t \wedge \tau_{n}}, t \in[0, T]$, a.s., $n=1,2, \ldots$ From here (19) follows as $n \rightarrow \infty$, because $\lim _{n \rightarrow \infty} \tau_{n}=\infty$ a.s.

44 
The notion of the differential generator of (12) is related to the Ito formula. To define it, introduce the matrix

$$
\left\|c_{i j}(t, \bar{x})\right\|_{i, j=1}^{m}=C(t, \bar{x})=B(t, \bar{x}) B(t, \bar{x})^{\prime}, \quad(t, \bar{x}) \in[0, T] \times R^{m},
$$

and set

$$
L=\frac{\partial}{\partial t}+\sum_{i=1}^{m} a^{i}(t, \bar{x}) \frac{\partial}{\partial x^{i}}+\frac{1}{2} \sum_{i, j=1}^{m} c_{i j}(t, \bar{x}) \frac{\partial^{2}}{\partial x^{i} \partial x^{j}},
$$

$L$ is called the differential generator associated with (12) (with the diffusion process having stochastic differential (12)). If (12) holds, and $f$ fulfils the assumptions of Theorem 4.2, then

$$
\mathrm{d} f\left(t, \bar{X}_{t}\right)=L f\left(t, \bar{X}_{t}\right) \mathrm{d} t+\left(\nabla f\left(t, \bar{X}_{t}\right)\right)^{\prime} B\left(t, \bar{X}_{t}\right) \mathrm{d} \bar{W}_{t}, \quad t \in[0, T],
$$

where $(\nabla f(t, \bar{x}))^{\prime}=\left(\partial f(t, \bar{x}) / \partial x^{1}, \ldots, \partial f(t, \bar{x}) / \partial x^{m}\right)$.

Example 2. The one-dimensional version of (12) is

$$
\mathrm{d} X_{t}=a\left(t, X_{t}\right) \mathrm{d} t+b\left(t, X_{t}\right) \mathrm{d} W_{t}, \quad t \in[0, T] .
$$

Consider the rather exceptional situation, when (20) with initial condition $X_{0} \equiv x_{0}$ is solvable in the form $u\left(t, W_{t}\right)$, where $u(t, y)$ is a sufficiently smooth function on $[0, T] \times(-\infty, \infty) . x_{0}$ is a number.

We have

$$
\mathrm{d} u\left(t, W_{t}\right)=\frac{\partial}{\partial t} u \mathrm{~d} t+\frac{\partial}{\partial y} u \mathrm{~d} W_{t}+\frac{1}{2} \frac{\partial^{2}}{\partial y^{2}} u \mathrm{~d} t
$$

Comparing (20) and (21) we deduce

$$
\frac{\partial}{\partial y} u=b(t, u), \quad(t, y) \in[0, T] \times(-\infty, \infty) .
$$

Further,

$$
\frac{\partial^{2}}{\partial y^{2}} u=\frac{\partial}{\partial x} b(t, u) \frac{\partial}{\partial y} u=b(t, u) \frac{\partial}{\partial x} b(t, u)
$$

Hence,

(23) $\quad \frac{\partial}{\partial t} u=a(t, u)-\frac{1}{2} b(t, u) \frac{\partial}{\partial x} b(t, u), \quad(t, y) \in[0, T] \times(-\infty, \infty)$.

We can thus assert the following: Let $b$ be continuous together with its first derivative with respect to $x$. Let function $u(t, y),(t, y) \in[0, T] \times(-\infty, \infty)$, have continuous 
derivatives $(\partial / \partial t) u,(\partial / \partial y) u$, and $\left(\partial^{2} / \partial y^{2}\right) u$. Let $u$ fulfil (22), (23), and $u(0,0)=x_{0}$. Then $X_{t}=u\left(t, W_{t}\right), t \in[0, T]$, is a solution of (20) with initial condition $X_{0} \equiv x_{0}$.

Under additional differentiability assumptions about $a, b$, equating $\left(\partial^{2} / \partial t \partial y\right) u$ and $\left(\partial^{2} / \partial y \partial t\right) u$, one gets from (22), (23) the necessary condition for their solvability:

$$
\frac{\partial}{\partial t} b+a \frac{\partial}{\partial x} b-b \frac{\partial}{\partial x} a+\frac{1}{2} b^{2} \frac{\partial^{2}}{\partial x^{2}} b=0 .
$$

Apply the above said to the equation

$$
\mathrm{d} X_{t}=a(t) X_{t} \mathrm{~d} t+b(t) X, \mathrm{~d} W_{t}, \quad t \in[0, T],
$$

where $a(t)$ and $b(t)$ are continuous on $[0, T] .(22),(23)$ are

$$
\frac{\partial}{\partial y} u=b(t) u, \quad \frac{\partial}{\partial t} u=a(t) u-\frac{1}{2} b(t)^{2} u
$$

Hence

$$
\begin{gathered}
u(t, y)=x_{0} \exp \left\{\int_{0}^{t}\left(a(s)-\frac{1}{2} b(s)^{2}\right) \mathrm{d} s+b(t) y\right\}, \\
(t, y) \in[0, T] \times(-\infty, \infty),
\end{gathered}
$$

or

$$
X_{t}=x_{0} \exp \left\{\int_{0}^{t}\left(a(s)-\frac{1}{2} b(s)^{2}\right) \mathrm{d} s+b(t) W_{t}\right\}, \quad t \in[0, T]
$$

\section{SUPPLEMENT}

\subsection{Random Variables on a Wiener Process}

The purpose of this last chapter is to add to the introduction into stochastic analysis an illustration of its more advanced parts. Two topics were selected. They are the representation of random variables defined on a Wiener process by means of stochastic integrals, and the Girsanov theorem. The former is important in filtering theory, the latter is a basic tool for studying the probability densities of diffusion processes.

Consider a probability space $(\Omega, \mathscr{A}, P)$, on which a Wiener process $W=\left\{W_{t}\right.$, $t \in[0, T]\}$ is defined. For the sake of simplicity assume $T<\infty$. Denote by $\mathscr{F}^{W}=$ $=\left\{\mathscr{F}_{t}^{W}, t \in[0, T]\right\}$ the increasing system of $\sigma$-algebras generated by $W$ and the $P$-null sets, i.e.

$$
\begin{gathered}
\mathscr{F}_{t}^{W}=\sigma a\left(\left\{W_{s} \leqq x\right\}, s \in[0, t], x \in(-\infty, \infty)\right. \\
P \text {-null sets }), \quad t \in[0, T]
\end{gathered}
$$


In this section we prove a theorem on representation of $\mathscr{F}_{T}^{W}$-measurable random variables. Nonanticipativity refers to $\mathscr{F}^{w}$.

Lemma 1. Let $\xi=g\left(W_{t_{t}}, \ldots, W_{t_{n}}\right)$, with $t_{0}=0<t_{1}<\ldots<t_{n} \leqq T$, and with function $g\left(x^{1}, \ldots, x^{n}\right)$ bounded, and having bounded continuous derivatives on $R^{n}$. Let $E \xi=0$. Then for a bounded nonanticipative function $\Phi=\left\{\Phi_{t}, t \in[0, T]\right\}$, it holds $\xi=\int_{0}^{T} \Phi \mathrm{d} W$.

Proof. Write

$$
\check{\zeta}=\sum_{i=0}^{n-1}\left(E\left\{\xi \mid \mathscr{F}_{t_{i+1}}^{W}\right\}-E\left\{\xi \mid \mathscr{F}_{t_{i}}^{W}\right\}\right)=\sum_{i=0}^{n-1} \int_{t_{i}}^{t_{i+1}} \mathrm{~d} E\left\{\xi \mid \mathscr{F}_{t}^{W}\right\} .
$$

Take an $i, 0 \leqq i \leqq n-1$. We shall show that $E\left\{\xi \mid \mathscr{F}_{t}^{W}\right\}$ has stochastic differential on $\left[t_{i}, t_{i+1}\right]$, and shall determine it. From the properties of the Wiener process it is seen that $E\left\{\xi \mid \mathscr{F}_{t}^{W}\right\}=\Psi\left(t, W_{t}\right)$, where

$$
\Psi(t, x)=\int \frac{1}{\sqrt{ }\left(2 \pi\left(t_{i+1}-t\right)\right)} \exp -\left[\frac{\left(x^{i+1}-x\right)^{2}}{2\left(t_{i+1}-t\right)}\right]\left\{\int \cdots \int g \left(W_{t_{1}}, \ldots,\right.\right.
$$

$$
\begin{gathered}
\left.W_{t_{1}}, x^{i+1}, \ldots, x^{n}\right) \cdot \frac{1}{\sqrt{\left(2 \pi\left(t_{i+2}-t_{i+1}\right)\right)} .} \\
\left.. \exp -\left[\frac{\left(x^{i+2}-x^{i+1}\right)^{2}}{2\left(t_{i+2}-t_{i+1}\right)}\right] \ldots \mathrm{d} x^{i+1} \ldots \mathrm{d} x^{n}\right\}, \\
(t, x) \in\left[t_{i}, t_{i+1}\right] \times(-\infty, \infty) .
\end{gathered}
$$

$\Psi(t, x)$ is a random function. However, since it is $\mathscr{F}_{t_{i}}^{W}$-measurable, Itô's formula can be used for $t \in\left[t_{i}, t_{i+1}\right]$. The first integrand in (1) is the fundamental solution of the heat equation. The hypotheses made about $g$ imply that $(\partial / \partial t) \Psi,\left(\partial^{2} / \partial x^{2}\right) \Psi$ are continuous on $\left[t_{i}, t_{i+1}\right] \times(-\infty, \infty)$, and that

$$
\frac{\partial}{\partial t} \Psi+\frac{1}{2} \frac{\partial^{2}}{\partial x^{2}} \Psi=0, \quad(t, x) \in\left[t_{i}, t_{i+1}\right] \times(-\infty, \infty) .
$$

Moreover, $(\partial / \partial x) \Psi$ is bounded. From the Itô formula we get

$$
\mathrm{d} \Psi\left(t, W_{t}\right)=\frac{\partial}{\partial t} \Psi \mathrm{d} t+\frac{\partial}{\partial x} \Psi \mathrm{d} W+\frac{1}{2} \frac{\partial^{2}}{\partial x^{2}} \Psi \mathrm{d} t=\frac{\partial}{\partial x} \Psi \mathrm{d} W
$$

Thus, we obtained the representation

$$
\xi=\sum_{i=0}^{n-1} \int_{t_{i}}^{t_{i+1}} \frac{\partial}{\partial x} \Psi \mathrm{d} W=\int_{0}^{T} \Phi \mathrm{d} W
$$


Theorem 1. Let $\xi$ be an $\mathscr{F}_{T}^{W}$-measurable random variable, $E \xi=0, E \xi^{2}<\infty$. Then there exists a nonanticipative function $\Phi=\left\{\Phi_{t}, t \in[0, T]\right\}$, satisfying $\int_{0}^{T} E \Phi^{2}$. . $\mathrm{d} t<\infty$, and such that $\xi=\int_{0}^{T} \Phi \mathrm{d} W$. $\Phi$ is determined uniquely up to $(t, \omega)$ from a null subset of $[0, T] \times \Omega$.

Proof. Since $\xi$ is $\mathscr{F}_{T}^{W}$-measurable, there exists a sequence $\left\{\xi_{n}, n=1,2, \ldots\right\}$ of random variables fulfilling the hypotheses of Lemma 1 such that 1.i.m. $\xi_{n}=\xi$.

By Lemma 1, $\xi_{n}=\int_{0}^{T n} \Phi \mathrm{d} W$ for nonanticipative ${ }^{n} \Phi, n=1,2, \ldots$ From

$$
0=\underset{\substack{m \rightarrow \infty \\ n \rightarrow \infty}}{\lim . \min } E\left(\xi_{m}-\xi_{n}\right)^{2}=\lim _{\substack{m \rightarrow \infty \\ n \rightarrow \infty}} \int_{0}^{T} E\left({ }^{m} \Phi-{ }^{n} \Phi\right)^{2} \mathrm{~d} t,
$$

and from the completeness property of the Hilbert space $L^{2}([0, T] \times \Omega)$ it follows that there exists a $\Phi$ such that

$$
\lim _{n \rightarrow \infty} \int_{0}^{T} E\left({ }^{n} \Phi-\Phi\right)^{2} \mathrm{~d} t=0
$$

$\Phi$ can be made nonanticipative. It follows from (2) that

$$
\xi=\underset{n \rightarrow \infty}{\operatorname{1in} .} \int_{0}^{T}{ }^{n} \Phi \mathrm{d} W=\int_{0}^{T} \Phi \mathrm{d} W .
$$

To verify the uniqueness, let also hold $\xi=\int_{0}^{T} \Phi^{*} \mathrm{~d} W, \int_{0}^{T} E \Phi^{* 2} \mathrm{~d} t<\infty$. Then $\int_{0}^{T}\left(\Phi^{*}-\Phi\right) \mathrm{d} W=0$ a.s. implies $\int_{0}^{T} E\left(\Phi^{*}-\Phi\right)^{2} \mathrm{~d} t=0$ as asserted in the theorem.

Assumption $\int_{0}^{T} E \Phi^{2} \mathrm{~d} t$ is needed to achieve unicity of $\Phi$, as it is seen from the next example.

Example 1. We shall construct a nonzero $\Phi=\left\{\Phi_{t}, t \in[0,1]\right\}$ such that $\int_{0}^{1} \Phi \mathrm{d} W=$ $=0$. Consider

$$
\bar{X}_{t}=\int_{0}^{t}(1-s)^{-1} \mathrm{~d} W_{s}, \quad t \in[0,1) .
$$

$X$ is a continuous Gaussian process with mean 0 and covariance

$$
\begin{gathered}
E X_{u} X_{t}=\int_{0}^{1} \chi_{\{s \leqq u\}} \chi_{\{s \leqq t\}}(1-s)^{-2} \mathrm{~d} s=\min \left\{u(1-u)^{-1}, t(1-t)^{-1}\right\}, \\
u, t \in[0,1) .
\end{gathered}
$$

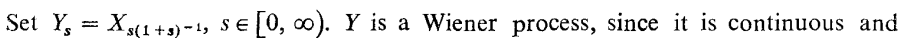
Gaussian with mean 0 and covariance $E Y_{u} Y_{s}=\min (u, s)$ for $u, s \in[0, \infty)$. Introduce the first passage times

$$
\sigma_{ \pm}=\inf \left\{s: Y_{s}= \pm 1\right\}
$$

From Example 2.2, it follows $0<\sigma_{ \pm}<\infty$ a.s.

48 
Define $\tau_{ \pm}=\sigma_{ \pm}\left(1+\sigma_{ \pm}\right)^{-1}$. Then $0<\tau_{ \pm}<1$ a.s., and

$$
\tau_{ \pm}=\inf \left\{t: \int_{0}^{t}(1-s)^{-1} \mathrm{~d} W_{s}= \pm 1\right\}
$$

Hence, $\tau_{ \pm}$are stopping times, and

$$
\Phi_{t}=\left(\chi_{\{t+>t\}}+\chi_{\left(r_{-}>t\right)}\right)(1-t)^{-1}, \quad t \in[0,1), \quad \Phi_{1} \equiv 0,
$$

is nonanticipative. We have

$$
\int_{0}^{1} \Phi_{s} \mathrm{~d} W_{s}=\int_{0}^{\tau_{+}}(1-s)^{-1} \mathrm{~d} W_{s}+\int_{0}^{\tau-}(1-s)^{-1} \mathrm{~d} W_{s}=0 \text { a.s. }
$$

Thus $\Phi$ has the desired property.

Under the assumptions of Theorem $2, E\left\{\xi \mid \mathscr{F}_{t}^{W}\right\}=\int_{0}^{t} \Phi \mathrm{d} W, t \in[0, T]$, in virtue of Property 3.3. Theorem 2 therefore provides representation of martingales with finite variance.

\subsection{Girsanov's Theorem}

Let on $(\Omega, \mathscr{A}, P)$ be defined a random process $X=\left\{X_{t}, t \in[0, T]\right\}, T<\infty$, such that it is the Wiener process with respect to $\mathscr{F}$. The hypothesis $X_{0} \equiv 0$ is not made. Further, let $\Phi=\left\{\Phi_{t}, t \in[0, T]\right\}$ be a nonanticipative function satisfying $\int_{0}^{T} \Phi^{2} \mathrm{~d} t<\infty$ a.s. Introduce

$$
Z_{t}=\exp \left\{\int_{0}^{t} \Phi \mathrm{d} X-\frac{1}{2} \int_{0}^{t} \Phi^{2} \mathrm{~d} s\right\}, \quad t \in[0, T],
$$

and assume

$$
E Z_{T}=1
$$

Recall the use of process $Z$ in $\S 3.1$, where it was shown that (3) is valid whenever $\Phi$ is a simple function.

Set $Z_{T}=\zeta$, and define probability measure $P^{*}$ on $(\Omega, \mathscr{A})$ by setting

$$
P^{*}(M)=\int_{M} \zeta \mathrm{d} P, \quad M \in \mathscr{A} .
$$

Process $X$ on probability space $\left(\Omega, \mathscr{A}, P^{*}\right)$ has the following property.

Theorem 2. (I. V. Girsanov) Let (3) hold. Then

$$
W=\left\{W_{t}=X_{t}-\int_{0}^{t} \Phi_{s} \mathrm{~d} s, \quad t \in[0, T]\right\}
$$

is a Wiener process on $\left(\Omega, \mathscr{A}, P^{*}\right)$. 
We postpone the proof to the end of this section. To explain the use of Theorem 2, let us consider the case, when $\Phi_{t}=a\left(t, X_{t}\right), t \in[0, T]$, where $a(t, x)$ is a Borel function on $[0, T] \times(-\infty, \infty)$. If $X$ is a Wiener process on $(\Omega, \mathscr{A}, P)$, then, according to Theorem 2 , it is on $\left(\Omega, \mathscr{A}, P^{*}\right)$ a diffusion process with stochastic differential

$$
\mathrm{d} X_{t}=a\left(t, X_{t}\right) \mathrm{d} t+\mathrm{d} W_{t}, \quad t \in[0, T] .
$$

Relation (4) thus states that a process with differential (5) has probability density with respect to the Wiener measure equal to

$$
\exp \left\{\int_{0}^{T} a\left(s, X_{s}\right) \mathrm{d} X_{s}-\frac{1}{2} \int_{0}^{T} a\left(s, X_{s}\right)^{2} \mathrm{~d} s\right\}
$$

For $a(t, x)$ constant, (6) can be obtained from a heuristic limit passage.

Example 2. Let $a$ be a constant, $W=\left\{W_{t}, t \in[0, T]\right\}, W_{0} \equiv 0$, a Wiener process. To derive the probability density of $X_{t}=a t+W_{t}, t \in[0, T]$, with respect to the Wiener measure, divide interval $[0, T]$ into $2^{n}$ subintervals of length $\Delta=2^{-n} T$. Random vectors

$$
\left(X_{\Delta}, X_{2 \Delta}, \ldots, X_{T}\right),\left(W_{\Delta}, W_{2 \Delta}, \ldots, W_{T}\right)
$$

have probability densities (with respect to the $2^{n}$-dimensional Lebesgue measure)

$$
p\left(x_{1}, \ldots, x_{j}, \ldots, x_{2^{n}}\right)=\frac{1}{(2 \pi \Delta)^{2^{n-1}}} \exp \left\{-\sum_{j=1}^{2^{n}} \frac{\left(x_{j}-x_{j-1}-a \Delta\right)^{2}}{2 \Delta}\right\},
$$

and

$$
p\left(x_{1}, \ldots, x_{j}, \ldots, x_{2^{n}}\right)=\frac{1}{(2 \pi \Delta)^{2^{n-1}}} \exp \left\{-\sum_{j=1}^{2^{n}} \frac{\left(x_{j}-x_{j-1}\right)^{2}}{2 \Delta}\right\}
$$

respectively. Their relative density is

$$
\frac{p\left(x_{1}, \ldots, x_{2^{n}}\right)}{p\left(x_{1}, \ldots, x_{2^{n}}\right)}=\exp \left\{\sum_{j=1}^{2^{n}}\left(\left(x_{j}-x_{j-1}\right) a-\frac{1}{2} a^{2} \Delta\right)\right\}=\exp \left\{a x_{2^{n}}-\frac{1}{2} a^{2} T\right\} .
$$

For $X_{T}=x_{2^{n}}$ this is (6) with $a(t, x) \equiv a$ independently of $n$. As $n \rightarrow \infty$, (7) passes into $\left\{X_{t}, t \in[0, T]\right\},\left\{W_{t}, t \in[0, T]\right\}$.

Next we give two lemmas.

Lemma 2. If (3) holds, then $Z$ is a martingale on $(\Omega, \mathscr{A}, P)$.

Proof. We have to verify

$$
E\left\{Z_{t}, \mid \mathscr{F}_{t}\right\}=Z_{t}, \quad 0 \leqq t \leqq t^{\prime} \leqq T,
$$


or

$$
\int_{A} Z_{t} \cdot \mathrm{d} P=\int_{A} Z_{t} \mathrm{~d} P, \quad A \in \mathscr{F}_{t}, \quad 0 \leqq t \leqq t^{\prime} \leqq T .
$$

Let $\left\{{ }^{n} \Phi, n=1,2, \ldots\right\}$ be a sequence of simple functions such that

$$
\int_{0}^{T}{ }^{n} \Phi^{2} \mathrm{~d} s<\infty \text { a.s., } \quad n=1,2, \ldots, \mathrm{p} \lim _{n \rightarrow \infty} \int_{0}^{T}\left({ }^{n} \Phi-\Phi\right)^{2} \mathrm{~d} s=0 .
$$

Define

$$
{ }^{n} Z_{u}=\exp \left\{\int_{0}^{u}{ }^{n} \Phi \mathrm{d} X-\frac{1}{2} \int_{0}^{u}{ }^{n} \Phi^{2} \mathrm{~d} s\right\}, \quad u \in[0, T], \quad n=1,2, \ldots
$$

By Property 3.5,

$$
\mathrm{p}_{n \rightarrow \infty} \lim ^{n} Z_{u}=Z_{u}, \quad u \in[0, T]
$$

By Lemma 3.1, " $Z, n=1,2, \ldots$, are martingales. Hence,

$$
E\left\{{ }^{n} Z_{t^{\prime}} \mid \mathscr{F}_{t}\right\}={ }^{n} Z_{t} \text { or } E\left\{{ }^{n} Z_{t^{\prime}}{ }^{n} Z_{t}^{-1} \mid \mathscr{F}_{t}\right\} \equiv 1 .
$$

From here we conclude that

$$
\int_{A}{ }^{n} Z_{t},{ }^{n} Z_{t}^{-1} Z_{t} \mathrm{~d} P=\int_{A} Z_{t} \mathrm{~d} P, \quad n=1,2, \ldots
$$

Letting $n \rightarrow \infty$, we obtain from (9) and from the Fatou lemma

$$
\int_{A} Z_{t^{\prime}} \mathrm{d} P \leqq \varliminf_{n \rightarrow \infty} \int_{A}{ }^{n} Z_{t^{\prime}}{ }^{n} Z_{t}^{-1} Z_{t} \mathrm{~d} P=\int_{A} Z_{t} \mathrm{~d} P .
$$

(10) shows that $Z$ is a supermartingale. To establish the martingale property, admit strict inequality in (10). Then

$$
\begin{gathered}
E Z_{T} \leqq E Z_{t^{\prime}}=\int_{\Omega-A} Z_{t^{\prime}} \mathrm{d} P+\int_{A} Z_{t^{\prime}} \mathrm{d} P<\int_{\Omega-A} Z_{t} \mathrm{~d} P+\int_{A} Z_{t} \mathrm{~d} P= \\
=E Z_{t} \leqq E Z_{0}=1 .
\end{gathered}
$$

This contradicts to (3). Hence, (8) holds.

Lemma 3. Let $\xi$ be a random variable, $E^{*}|\xi|=E|\xi| \zeta<\infty$. Then

$$
E^{*}\left\{\zeta \mid \mathscr{F}_{t}\right\}=Z_{t}^{-1} E\left\{\xi \zeta \mid \mathscr{F}_{t}\right\}, \quad t \in[0, T] .
$$


Proof. We have to prove

$$
\int_{A} \xi \mathrm{d} P^{*}=\int_{A} Z_{t}^{-1} E\left\{\xi \zeta \mid \mathscr{F}_{t}\right\} \mathrm{d} P^{*}, \quad A \in \mathscr{F}_{t}, \quad t \in[0, T]
$$

Lemma 1 implies for $M \in \mathscr{F}$,

$$
P^{*}(M)=\int_{M} E\left\{\zeta \mid \mathscr{F}_{t}\right\} \mathrm{d} P=\int_{M} Z_{t} \mathrm{~d} P
$$

Hence, because the integrand on the right-hand side of (12) is $\mathscr{F}_{t}$-measurable, the integral there equals

$$
\int_{A} Z_{t}^{-1} E\left\{\zeta \zeta \mid \mathscr{F}_{t}\right\} Z_{t} \mathrm{~d} P=\int_{A} \xi \zeta \mathrm{d} P=\int_{A} \xi \mathrm{d} P^{*}
$$

Proof of Theorem 2. The proof is similar to that of Theorem 4.3. We shall demonstrate for $0 \leqq t<t^{\prime} \leqq T$

$$
\begin{gathered}
E^{*}\left\{\exp \left\{\mathrm{i} z\left(X_{t^{\prime}}-X_{t}-\int_{t}^{t^{\prime}} \Phi \mathrm{d} s\right)\right\} \mid \mathscr{F}_{t}\right\}=\exp \left\{-\frac{1}{2} z^{2}\left(t^{\prime}-t\right)\right\}, \\
z \in(-\infty, \infty) .
\end{gathered}
$$

Using Lemma 3 and the properties of conditional expectations we conclude that the left-hand side of (13) equals

$$
E\left\{\exp \left\{\mathrm{i} z\left(X_{t^{\prime}}-X_{t}-\int_{t}^{t^{\prime}} \Phi \mathrm{d} s\right)\right\} E\left\{Z_{T} \mid \mathscr{F}_{t^{\prime}}\right\} Z_{t}^{-1} \mid \mathscr{F}_{t}\right\}=E\left\{Y_{t^{\prime}} \mid \mathscr{F}_{t}\right\},
$$

where

$$
Y_{u}=\exp \left\{\mathrm{i} z\left(X_{u}-X_{t}-\int_{t}^{u} \Phi \mathrm{d} s\right)+\int_{t}^{u} \Phi \mathrm{d} X-\frac{1}{2} \int_{t}^{u} \Phi^{2} \mathrm{~d} s\right\}, \quad u \in[t, T] .
$$

The above stated is true also, if we replace $t^{\prime}$ by a stopping time $\tau, t \leqq \tau \leqq T$. Namely, it holds

$$
E^{*}\left\{\exp \left\{\mathrm{i} z\left(X_{\tau}-X_{t}-\int_{t}^{\tau} \Phi \mathrm{d} s\right)\right\} \mid \mathscr{F}_{t}\right\}=E\left\{Y_{\tau} \mid \mathscr{F}_{t}\right\} .
$$

$Y$ has stochastic differential

$$
\begin{gathered}
\mathrm{d} Y=Y\left(\mathrm{i} z(\mathrm{~d} X-\Phi \mathrm{d} u)+\Phi \mathrm{d} X-\frac{1}{2} \Phi^{2} \mathrm{~d} u\right)+\frac{1}{2} Y\left(-z^{2} \mathrm{~d} u+2 \mathrm{i} z \Phi \mathrm{d} u+\Phi^{2} \mathrm{~d} u\right)= \\
=(\mathrm{i} z+\Phi) Y \mathrm{~d} X-\frac{1}{2} z^{2} Y \mathrm{~d} u
\end{gathered}
$$


The integral of the before last term has expectation zero provided that Property 3.3 is valid. To achieve it introduce stopping times

$$
\tau_{n}=\inf \left\{u: \int_{t}^{u}|(\mathrm{i} z+\Phi) Y|^{2} \mathrm{~d} s \geqq n\right\} \wedge t^{\prime}, \quad n=1,2, \ldots
$$

Then, by Property 3.7

$$
Y_{t_{n}}-1=\int_{t}^{t^{r}} \chi_{\left\{\tau_{n}>u\right\}}(\mathrm{i} z+\Phi) Y \mathrm{~d} X-\frac{1}{2} z^{2} \int_{t}^{\tau_{n}} Y \mathrm{~d} u .
$$

Property 3.3 implies that the conditional expectation of the first right-hand side term vanishes. Therefore,

$$
\begin{gathered}
E^{*}\left\{\exp \left\{\mathrm{i} z\left(X_{\tau_{n}}-X_{t}-\int_{t}^{\tau_{n}} \Phi \mathrm{d} s\right)\right\} \mid \mathscr{F}_{t}\right\}=E\left\{Y_{\tau_{n}} \mid \mathscr{F}_{t}\right\}= \\
=1-\frac{1}{2} z^{2} E\left\{\int_{t}^{\tau_{n}} Y \mathrm{~d} u \mid \mathscr{F}_{t}\right\} .
\end{gathered}
$$

For $n=1,2, \ldots$, the random variable in the first conditional expectation in (14) is bounded. The last random variable has an integrable majorant, since

$$
\left|\int_{t}^{\tau_{n}} Y \mathrm{~d} u\right| \leqq \int_{t}^{t^{\prime}} Z_{u} Z_{t}^{-1} \mathrm{~d} u, \quad E \int_{t}^{t^{\prime}} Z_{u} Z_{t}^{-1} \mathrm{~d} u=\int_{t}^{t^{\prime}} E Z_{u} Z_{t}^{-1} \mathrm{~d} u=t^{\prime}-t .
$$

Furthermore,

$$
\tau_{n} \leqq \tau_{n+1}, \quad n=1,2, \ldots, \lim _{n \rightarrow \infty} \tau_{n}=t^{\prime} \quad \text { a.s. }
$$

Letting $n \rightarrow \infty$ in (14), we thus obtain,

$$
\begin{aligned}
& E^{*}\left\{\exp \left\{\mathrm{i} z\left(X_{i^{\prime}}-X_{t}-\int_{t}^{t^{\prime}} \Phi \mathrm{d} s\right)\right\} \mid \mathscr{F}_{t}\right\}=1-\frac{1}{2} z^{2} \int_{t}^{t^{\prime}} E\left\{Y_{u} \mid \mathscr{F}_{t}\right\} \mathrm{d} u= \\
& =1-\frac{1}{2} z^{2} \int_{t}^{t^{\prime}} E^{*}\left\{\exp \left\{\mathrm{i} z\left(X_{u}-X_{t}-\int_{t}^{u} \Phi \mathrm{d} s\right)\right\} \mid \mathscr{F}_{t}\right\} \mathrm{d} u, \quad t^{\prime} \in[t, T] .
\end{aligned}
$$

This is an integral equation, the only solution of which is given by (13). 
[1] М. Арато, А. Н. Колмогоров, Я. Г. Синай: Об оңенке параметров комплексного стационарного гауссовского-марковското процесса. Доклады АН СССР 146 (1962), 430-435.

[2] K. J. Åström: Introduction to Stochastic Control Theory. Academic Press, New York-London 1970.

[3] J. M. C. Clark: The representation of functionals of Brownian motion by stochastic integrals. Annals of Math. Stat. 41 (1970), 1282-1295.

[4] J. L. Doob: Stochastic Processes. J. Wiley, New York-London 1953.

[5] И. И. Гихман, А. В.Скороход: Теория случайных процессов, том III. Наука, Москва 1975.

[6] Р. Ш. Липцер, А. Н. Ширяев: Статистика случайных процессов. Наука, Москва 1974.

[7] P. Mandl: Stochastic Integrals and their Applications. (In Czech.) Inst. of Information Theory and Automation, Czechosl. Acad. Sc., Prague 1976.

[8] H. P. Mc Kean, Jr.: Stochastic Integrals. Academic Press, New York-London 1969.

[9] P. A. P. Moran: An Introduction to Probability Theory. Clarendon Press, Oxford 1968.

[10] M. Rosenblatt: Random Processes. Springer-Verlag, New York-Heidelberg-Berlin 1974. 